\title{
Dissecting trait heterogeneity: a comparison of three clustering methods applied to genotypic data Tricia A Thornton-Wells ${ }^{1,2,3}$, Jason H Moore ${ }^{4}$ and Jonathan L Haines*3,5
}

\begin{abstract}
Address: ${ }^{1}$ Neuroscience Graduate Program, Vanderbilt Brain Institute, Vanderbilt University Medical Center, Nashville, TN, USA, ${ }^{2}$ Department of Biomedical Informatics, Vanderbilt University Medical Center, Nashville, TN, USA, ${ }^{3}$ Center for Human Genetics Research, Vanderbilt University Medical Center, Nashville, TN, USA, ${ }^{4}$ Computational Genetics Laboratory, Department of Genetics, Dartmouth Medical School, Lebanon, NH, USA and ${ }^{5}$ Department of Molecular Physiology and Biophysics, Vanderbilt University Medical Center, Nashville, TN, USA
\end{abstract}

Email: Tricia A Thornton-Wells - t.thornton-wells@vanderbilt.edu; Jason H Moore - jason.h.moore@dartmouth.edu; Jonathan L Haines* - jonathan@chgr.mc.vanderbilt.edu

* Corresponding author

Published: 12 April 2006

BMC Bioinformatics 2006, 7:204 doi:10.1186/147/-2105-7-204
Received: 08 September 2005

Accepted: 12 April 2006

This article is available from: http://www.biomedcentral.com/I47I-2/05/7/204

(C) 2006 Thornton-Wells et al; licensee BioMed Central Ltd.

This is an Open Access article distributed under the terms of the Creative Commons Attribution License (http://creativecommons.org/licenses/by/2.0), which permits unrestricted use, distribution, and reproduction in any medium, provided the original work is properly cited.

\begin{abstract}
Background: Trait heterogeneity, which exists when a trait has been defined with insufficient specificity such that it is actually two or more distinct traits, has been implicated as a confounding factor in traditional statistical genetics of complex human disease. In the absence of detailed phenotypic data collected consistently in combination with genetic data, unsupervised computational methodologies offer the potential for discovering underlying trait heterogeneity. The performance of three such methods Bayesian Classification, Hypergraph-Based Clustering, and Fuzzy k-Modes Clustering - appropriate for categorical data were compared. Also tested was the ability of these methods to detect trait heterogeneity in the presence of locus heterogeneity and/or gene-gene interaction, which are two other complicating factors in discovering genetic models of complex human disease. To determine the efficacy of applying the Bayesian Classification method to real data, the reliability of its internal clustering metrics at finding good clusterings was evaluated using permutation testing.

Results: Bayesian Classification outperformed the other two methods, with the exception that the Fuzzy $k$-Modes Clustering performed best on the most complex genetic model. Bayesian Classification achieved excellent recovery for $75 \%$ of the datasets simulated under the simplest genetic model, while it achieved moderate recovery for $56 \%$ of datasets with a sample size of 500 or more (across all simulated models) and for $86 \%$ of datasets with 10 or fewer nonfunctional loci (across all simulated models). Neither Hypergraph Clustering nor Fuzzy k-Modes Clustering achieved good or excellent cluster recovery for a majority of datasets even under a restricted set of conditions. When using the average log of class strength as the internal clustering metric, the false positive rate was controlled very well, at three percent or less for all three significance levels $(0.0 \mathrm{I}, 0.05,0.10)$, and the false negative rate was acceptably low (I8 percent) for the least stringent significance level of 0.10 .

Conclusion: Bayesian Classification shows promise as an unsupervised computational method for dissecting trait heterogeneity in genotypic data. Its control of false positive and false negative rates lends confidence to the validity of its results. Further investigation of how different parameter settings may improve the performance of Bayesian Classification, especially under more complex genetic models, is ongoing.
\end{abstract}




\begin{tabular}{|c|c|c|c|}
\hline & Locus Heterogeneity & Trait Heterogeneity & Gene-Gene Interaction \\
\hline Definition & $\begin{array}{l}\text { when two or more DNA variations in } \\
\text { distinct genetic loci are independently } \\
\text { associated with the same trait }\end{array}$ & $\begin{array}{l}\text { when a trait, or disease, has been defined } \\
\text { with insufficient specificity such that it is } \\
\text { actually two or more distinct underlying traits }\end{array}$ & $\begin{array}{l}\text { when two or more DNA variations interact } \\
\text { either directly (DNA-DNA or DNA-mRNA } \\
\text { interactions), to change transcription or } \\
\text { translation levels, or indirectly by way of } \\
\text { their protein products, to alter disease risk } \\
\text { separate from their independent effects }\end{array}$ \\
\hline Diagram & $\begin{array}{l}\text { Allelic Variant } \mathrm{i} \\
\text { Of Locus } \mathrm{A}\end{array}$ & $\searrow_{\text {Disease } \mathrm{X}}^{\text {Trait I }}$ & Allelic Variant i \\
\hline $\begin{array}{l}\text { Example } \\
\text { One }\end{array}$ & $\begin{array}{l}\text { Retinitis Pigmentosa (RP, OMIM\# } \\
268000 \text { ) - genetic variations in at least } \\
\text { fifteen genes have been associated } \\
\text { with RP under an autosomal recessive } \\
\text { model. Still more have been } \\
\text { associated with RP under autosomal } \\
\text { dominant and X-linked disease } \\
\text { models }{ }^{2} \\
\text { (http://www.sph.uth.tmc.edu/RetNet) }\end{array}$ & $\begin{array}{l}\text { Autosomal Dominant Cerebellar Ataxia } \\
\text { (ADCA, OMIM\# 164500) - originally } \\
\text { described as a single disease, three different } \\
\text { clinical subtypes have been defined based } \\
\text { on variable associated symptoms, }{ }^{6,7} \text { and } \\
\text { different genetic loci have been associated } \\
\text { with the different subtypes }\end{array}$ & $\begin{array}{l}\text { Hirschsprung Disease (OMIM\# 142623) - } \\
\text { variants in the RET (OMIM\# 164761) and } \\
\text { EDNRB (OMIM\# 131244) genes have } \\
\text { been shown to interact synergistically such } \\
\text { that they increase disease risk far beyond } \\
\text { the combined risk of the independent } \\
\text { variants }^{12}\end{array}$ \\
\hline $\begin{array}{l}\text { Example } \\
\text { Two }\end{array}$ & $\begin{array}{l}\text { Tuberous Sclerosis (TS, OMIM\# } \\
\text { 191100) - out of families informative for } \\
\text { linkage analysis, half have mutations in } \\
\text { the TSC1 gene (located at 9q34) and } \\
\text { the other half have mutations in the } \\
\text { TSC2 gene (located at 16p13) } \\
\text { 3.,4,5 }\end{array}$ & $\begin{array}{l}\text { Autism (OMIM\# 209850) - parents and other } \\
\text { relatives of autistic individuals often exhibit } \\
\text { one or two, but not all three, of the requisite } \\
\text { autistic symptomatologies, suggesting } \\
\text { autism may be the co-occurrence of three } \\
\text { distinct traits. }{ }^{9} \text { Using subset analysis, some } \\
\text { success has been achieved identifying } \\
\text { genes associated with one of the three } \\
\text { symptomatologies but not as strongly with } \\
\text { the broader autistic phenotype }\end{array}$ & $\begin{array}{l}\text { Creutzfeldt-Jakob Disease (CJD, OMIM\# } \\
123400 \text { ) and Fatal Familial Insomnia } \\
\text { (OMIM\# 176640.0010) - the Met129Val } \\
\text { polymorphism and Asp178Asn mutation in } \\
\text { the PRNP gene (OMIM\# 176640) interact, } \\
\text { such that when the val129 polymorphism } \\
\text { is on the same chromosome as the } \\
\text { asn178, the phenotype is fatal familial } \\
\text { insomnia } 13-19\end{array}$ \\
\hline
\end{tabular}

Figure I

Factors Complicating Analysis of Complex Genetic Disease: Definitions, Diagrams and Examples

\section{Background}

\section{Complex human genetic disease}

Molecular biologists and geneticists alike now acknowledge that the most common human diseases with a genetic component are likely to have very complex etiologies, involving such complicating factors as locus heterogeneity, trait heterogeneity, and gene-gene interactions (Figure $1 ;[1-19])$. However, only a small fraction of the human genetics literature specifically reports on investigations of such complexity. Statistical geneticists continue primarily using traditional methodologies, such as linkage and association, which often detect but fail to replicate findings of main effect genes. While undoubtedly many of the original positive results are false-positives, true effects may not be replicated for many reasons, including population stratification and true differences in genetic etiology between study populations [20].

Current statistical approaches to detecting heterogeneity, such as the admixture test $[21,22]$, are neither sensitive nor powerful and can merely account for, not resolve, any underlying heterogeneity. In addition, while a small number of supervised computational methods exist for discovering gene-gene interactions, the power of these methods drops dramatically when locus or trait heterogeneity is present $[23,24]$. Phenotypic data can be utilized to improve the performance of these methods in the face of locus or trait heterogeneity by facilitating heuristic stratification of data. However, for many diseases, little detailed phenotypic data has been collected consistently in combination with genotypic data. It is for these reasons that an unsupervised method, which does not rely on phenotypic data, is needed to mine potentially heterogeneous genotypic data as a means of data stratification and hypothesis generation.

For genetic factors involving heterogeneity, there are multiple independent (predictor) variables or else multiple dependent (outcome) variables that complicate the analysis by creating a heterogeneous model landscape. In the case of locus heterogeneity, multiple predictor variables (i.e., multiple loci) are present, some of which may be unmeasured or unobserved and, therefore, unavailable 
Table I: Confidence intervals around $A R I_{H A}$ means by method.

\begin{tabular}{lcccc}
\hline & & & \multicolumn{2}{c}{$95 \%$ Confidence Interval } \\
\hline Method & Mean & $\begin{array}{c}\text { Standard } \\
\text { Error }\end{array}$ & Lower End & Upper End \\
Bayesian & 0.666 & 0.001 & 0.664 & 0.667 \\
Hypergraph & 0.354 & 0.001 & 0.352 & 0.355 \\
Fuzzy k-Modes & 0.556 & 0.001 & 0.555 & 0.558 \\
\hline
\end{tabular}

for inclusion in the disease model. In the case of trait heterogeneity, multiple outcome variables are present, which cannot or have not been distinguished based on the available phenotypic information. Gene-gene interactions create a rugged model landscape for statistical analysis. There is clear and convincing evidence that gene-gene interactions, whether synergistic or antagonistic, are not only possible but probably ubiquitous $[18,19,25-28]$. Thus, it is critical that complex genetic data sets be properly interrogated for possible underlying interactions.

\section{Statistical analysis}

No one analytic method is superior in all respects for the range of complicating factors that might be present in a specific data set. Given the relative shortcomings of our current analyses in complex diseases, we need to greatly extend the range of available analytical tools. There is a critical need for extensive reevaluation of existing methodologies for complex diseases, as well as for massive efforts in new method development. It is important that empirical studies be conducted to compare and contrast the relative strengths and weaknesses of methods on specific types of problems. For example, while cluster analysis has shown promise in numerous scientific and mathematical fields, including genetics $[29,30]$, its use with discrete genotypic data has not been adequately explored. Similarly, artificial neural networks modified with evolutionary computation have great potential for discovering nonlinear interactions among genes and environmental factors [31]. However, work is still ongoing to evaluate their limitations with regard to the heritability and effect sizes that can be detected.

Ultimately, though, the real power of existing and yet-tobe-developed methods lies in our ability to marry them into a comprehensive approach to genetic analysis, so that their relative strengths and weaknesses can be balanced and few alternative hypotheses are left uninvestigated. Because no single method adequately investigates heterogeneity and interaction issues simultaneously, we propose routinely taking a two-step approach to analysis. For example, clustering or ordered subset analysis [32] can be used first to uncover genotypic and/or phenotypic heterogeneity and to subdivide the data into more homogeneous groups. Then in a second step, specific tests of interactions, such as the $\mathrm{S}$ sum statistic approach $[33,34,34]$ or the multifactor dimensionality reduction method [23,24,35-37] could be used to investigate genegene or gene-environment interactions within each of the homogenized subgroups. While this is not a perfect approach, it is an important improvement over the more common alternative of a single-pronged approach to analysis.

\section{Cluster analysis}

For over 30 years, cluster analysis has been used as a method of data exploration [38]. Clustering is an unsupervised classification methodology, which attempts to uncover 'natural' clusters or partitions of data. It involves data encoding and choosing a similarity metric, which will be used in determining the relative 'goodness' of a clustering of data. No one clustering method has been shown universally effective when applied to the wide variety of structures present in multidimensional data sets. Instead, the choice of suitable methods is dependent on the type of target data to be analyzed. Clustering has been utilized widely for the analysis of gene expression (e.g., DNA microarray) data; however, its application to genotypic data has been limited [29].

Most traditional clustering algorithms use a similarity metric based on distance that may be inappropriate for categorical data such as genotypes. Newer methods have been developed with categorical data in mind and include extensions of traditional methods and application of probabilistic theory. Three such methods were chosen for comparison in the task of discovering trait heterogeneity using multilocus genotypes - Bayesian Classification [39], Hypergraph-Based Clustering [40], and Fuzzy $k$-Modes Clustering [41] - all of which are appropriate for categorical data.

\section{Results}

\section{Descriptive statistics}

The Hubert-Arabie Adjusted Rand Index (ARI ${ }_{\mathrm{HA}}$ ) was chosen as the standard for measuring clustering result fitness [42]. An ARI $\mathrm{HA}_{\mathrm{HA}}$ score of 0.90 indicates excellent cluster recovery, 0.80 good recovery, and 0.65 moderate recovery. Mean $\mathrm{ARI}_{\mathrm{HA}}$ values for Bayesian Classification, Hypergraph Clustering and Fuzzy $k$-Modes Clustering were $0.666,0.354$ and 0.556 , respectively. Confidence intervals around the means were also produced to demonstrate the preciseness of the $\mathrm{ARI}_{\mathrm{HA}}$ measurements. The results for each method across all datasets are presented in Table 1. Mean $\mathrm{ARI}_{\mathrm{HA}}$ values differed by genetic model type (Figure 2 ), with higher scores achieved on Trait Heterogeneity Only (THO) datasets for the Bayesian Classification and Hypergraph Clustering methods (Figure 3). 


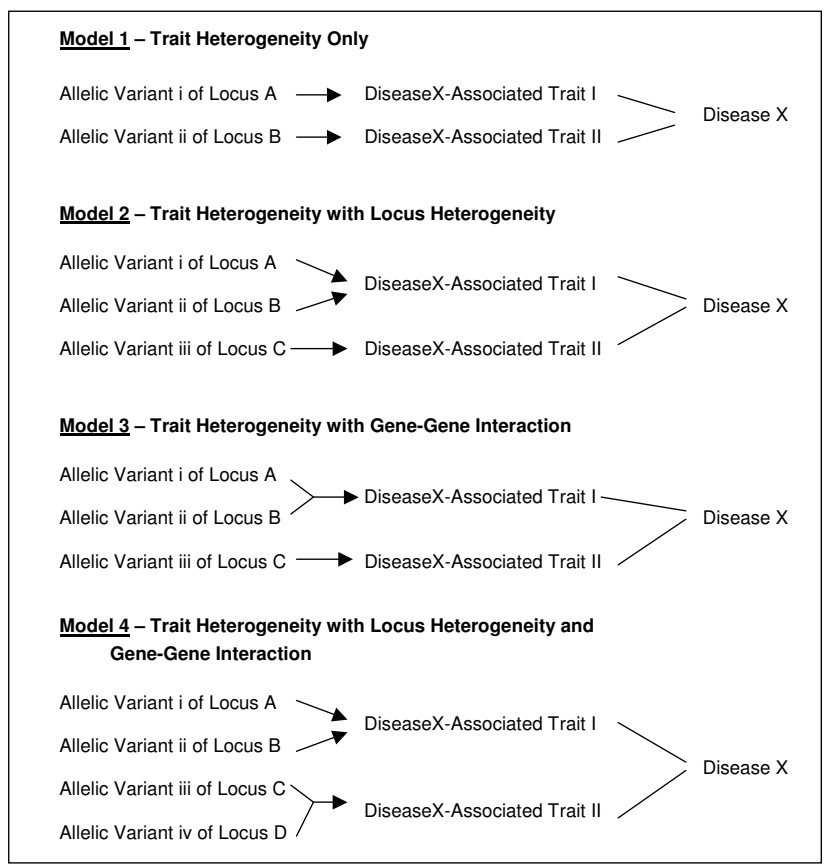

Figure 2

Structure of Genetic Models Used for Data Simulation

\section{Comparison of clustering methods}

Three categorical variables were constructed that could be tested using the nonparametric chi-square test of independence. The three variables were calculated as the number of clustering results achieving each of the three $\mathrm{ARI}_{\mathrm{HA}}$ cutoff values of 0.90 (for excellent cluster recovery), 0.80 (for good cluster recovery) and 0.65 (for moderate cluster recovery). Results are displayed as percentages by clustering method (Figure 4) and by clustering method and genetic model (Figure 5A). A chi-square test of independence was performed testing the null hypothesis that the number of clusterings achieving the specified $\mathrm{ARI}_{\mathrm{HA}}$ cutoff value was independent of the clustering method. The three methods performed significantly differently on each of the $\mathrm{ARI}_{\mathrm{HA}}$ cutoff statistics (Table 2). Bayesian Classification outperformed the other two methods. However, across all the dataset parameters, Bayesian Classification achieved moderate or better recovery on only $48 \%$ of the datasets (Figure 4).

The performance of the three clustering methods across different dataset parameters was evaluated in an attempt to find particular conditions under which one method consistently achieved good or excellent recovery (not just better recovery than the other two methods). For those datasets simulated under the THO model, Bayesian Classification performed well, with over 73 percent of its resulting clusterings achieving an ARIHA value of 0.90 or greater, indicating excellent recovery (Figure 5). For this

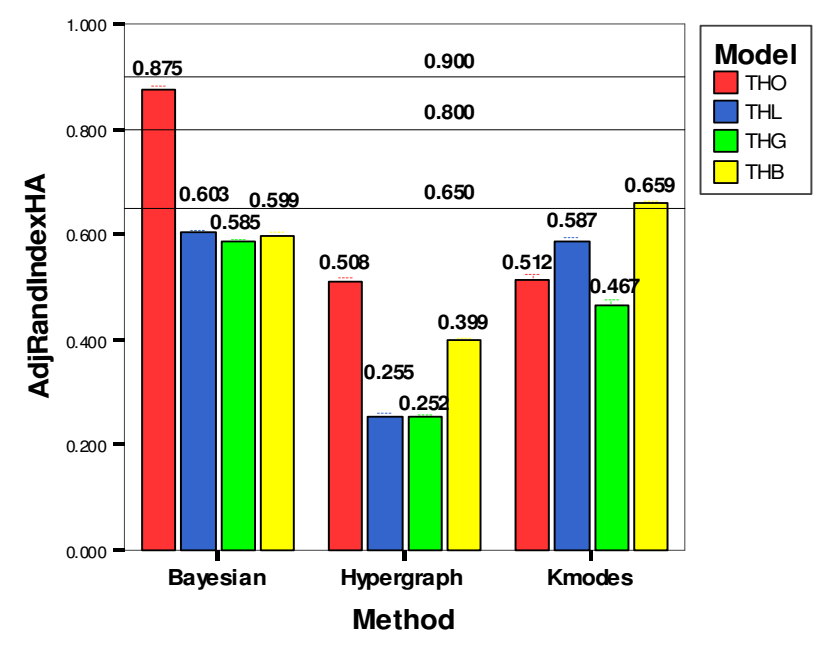

\section{Figure 3}

Comparison of $\mathrm{ARI}_{\mathrm{HA}}$ Means by Method and Model. Comparison of Hubert-Arabie Adjusted Rand Index means by method and model. Bars represent means, and error bars, which are very short and may be difficult to see, represent $95 \%$ confidence intervals. Horizontal lines represent thresholds for quality of cluster recovery: 0.90 for excellent recovery, 0.80 for good recovery and 0.65 for moderate recovery.

subset of the datasets, Bayesian Classification outperformed the other two methods, and again there was a significant difference in performance across the three methods, as measured by a chi-square test of independence on each of the three new ARIHA cutoff statistics (Table 3). Analysis of the other simulation parameters failed to show as great a difference among methods where the 'winning' method performed as well as the Bayesian Classification performed in the THO datasets (data not shown). Thus, this subset of data was chosen for further investigation into the efficacy of using the Bayesian Classification method to uncover trait heterogeneity in real data.

\section{Applicability to real data}

To evaluate the validity of using the Bayesian Classification internal clustering metrics - class strength and crossclass entropy - as a proxy for the $A_{\mathrm{HA}}$ (since $A R I_{\mathrm{HA}}$ is unknown for real data), permutation testing was performed. Resulting p-values for $\mathrm{ARI}_{\mathrm{HA}^{\prime}}$ average log of class strength and average cross class entropy were used to calculate false positive and false negative rates at three significance levels of $0.01,0.05$ and 0.10 . A clustering result was considered a false positive if it was considered significant according to either average log of class strength or average cross class entropy but was not considered significant according to our $\mathrm{ARI}_{\mathrm{HA}}$ standard. A clustering result was considered a false negative if it was called not-significant according to both average log of class strength and aver- 


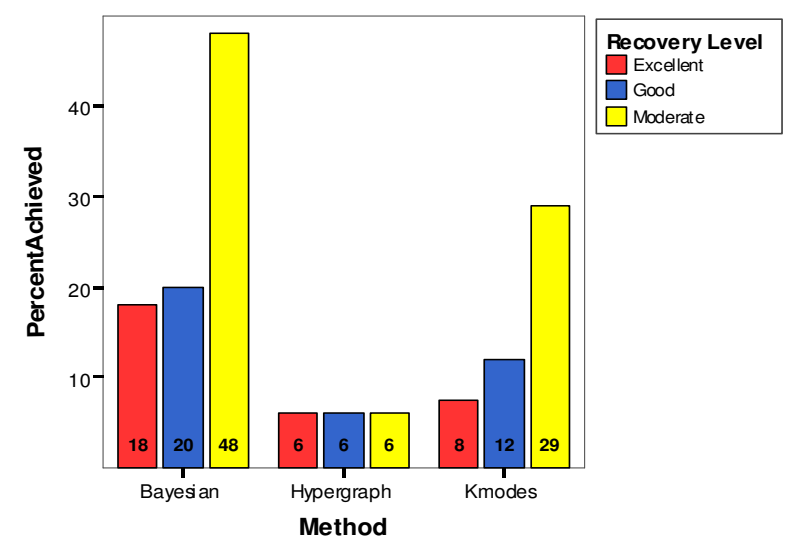

Figure 4

Percentage of Clustering Results Achieving Cluster Recovery Levels by Method

age cross class entropy but was considered significant according to $\mathrm{ARI}_{\mathrm{HA}}$. Figures 6 and 7 show the false positive and false negative rates, respectively, by alpha level.

The false positive, or Type I, error rate was controlled very well at three percent or less for all three significance levels. The false negative, or Type II, error rate was not controlled as well, however. At the least stringent significance level ( $\alpha$ $=0.10)$, the Type II error rate was 18 percent, and at the most stringent level $(\alpha=0.01)$, the rate was 47 percent. Other simulation parameters were examined for their impact on the false negative rate, and Figures 8 and 9 show the false negative rate by alpha level paneled by number of nonfunctional loci and number of affecteds (sample size), respectively. As might be expected, the lowest false negative rates were achieved for datasets with the lowest number of nonfunctional loci (10) and the greatest sample size (1000).

\section{Discussion}

Data simulation

The new data simulation algorithm produced complex genotypic datasets that included trait heterogeneity, locus heterogeneity and gene-gene interactions. Most existing simulation software that attempt to simulate heterogeneity do so by allowing the user to specify what portion of the dataset is to be simulated under one model versus another, and the resulting individuals are simply combined into one dataset. In the new algorithm, however, the disease penetrance models, which were used to simulate the data, were constructed so that overall prevalence levels were controlled, allowing naturally occurring overlaps, in which some individuals would have both traits (and their associated multilocus genotypes) by chance. This novel data simulation algorithm should prove very
Table 2: Overall results of Chi-Square Test of Independence. Overall results of chi-square test of independence testing the null hypothesis that the percentage of clustering results achieving the specified cluster recovery level does not differ across clustering methods.

\begin{tabular}{|c|c|c|c|}
\hline Cluster Recovery Statistic & $\chi^{2}$ & df & $\mathbf{p}$ \\
\hline $\begin{array}{l}\text { \%Results achieving Excellent cluster recovery } \\
\left(A R I_{H A} \geq 0.90\right)\end{array}$ & 1787 & 2 & $<0.001$ \\
\hline $\begin{array}{l}\text { \%Results achieving Good cluster recovery } \\
\left(A R I_{H A} \geq 0.80\right)\end{array}$ & 1614 & 2 & $<0.001$ \\
\hline $\begin{array}{l}\text { \%Results achieving Moderate cluster recovery } \\
\left(A R I_{H A} \geq 0.65\right)\end{array}$ & 8565 & 2 & $<0.001$ \\
\hline
\end{tabular}

useful for future studies of other proposed genetic analysis methods for complex diseases.

\section{Comparison of clustering methods}

The Bayesian Classification method outperformed the other two methods across most dataset parameter combinations, with the exception of the most complex model (THB) on which Fuzzy $k$-Modes Clustering performed best. When the results were further examined to find a set of parameters for which one or more methods performed well, Bayesian Classification was found to have achieved excellent recovery for $73 \%$ of the datasets with the THO model and achieved moderate recovery for $56 \%$ of datasets with 500 or more affecteds and for $86 \%$ of datasets with 10 or fewer nonfunctional loci (data not shown here). Neither Hypergraph Clustering nor Fuzzy $k$-Modes Clustering achieved good or excellent cluster recovery even under a restricted set of conditions.

Bayesian Classification was obtained as closed-source software, for which there were numerous parameters, which could have been optimized. Initial parameter settings were chosen as recommended by the authors based on the type of data being analyzed. However, it is possible that alternative settings may have yielded better results. For example, for datasets with the more complex genetic models, greater numbers of nonfunctional loci and smaller sample sizes, the maximum number of classifica-

Table 3: Results of Chi-Square Test of Independence for Trait Heterogeneity Only datasets. Results of chi-square test of independence for Trait Heterogeneity Only datasets, testing the null hypothesis that the percentage of clustering results achieving the specified cluster recovery level does not differ across clustering methods.

\begin{tabular}{lcccc}
\hline Cluster Recovery Statistic & Model & $\chi^{2}$ & df & p \\
\hline $\begin{array}{l}\text { \%Results achieving Excellent cluster } \\
\text { recovery }\left(A R I_{\mathrm{HA}} \geq 0.90\right)\end{array}$ & THO & 3713 & 2 & $<0.001$ \\
$\begin{array}{l}\text { \%Results achieving Good cluster } \\
\text { recovery }\left(\mathrm{ARI} \mathrm{HA}_{\mathrm{HA}} \geq 0.80\right)\end{array}$ & THO & 3107 & 2 & $<0.001$ \\
$\begin{array}{l}\text { \%Results achieving Moderate cluster } \\
\text { recovery }\left(\mathrm{ARI} \mathrm{HA}_{\mathrm{HA}} \geq 0.65\right)\end{array}$ & THO & 2609 & 2 & $<0.00 \mathrm{I}$ \\
\hline
\end{tabular}



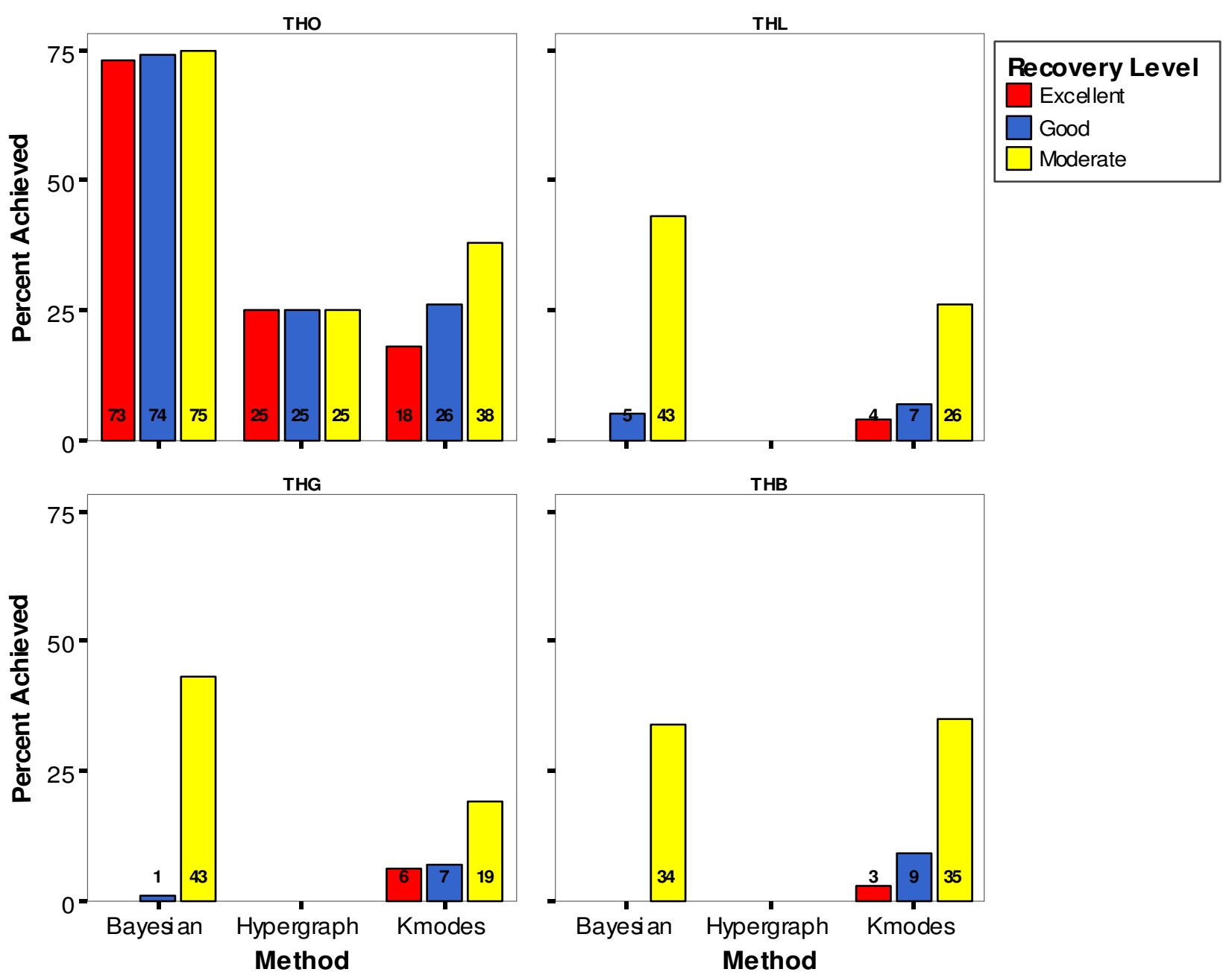

\section{Figure 5}

Percentage of Clustering Results Achieving Cluster Recovery Levels by Method and Model

tion trials and/or the maximum number of classification cycles per trial may need to be longer, and those parameters concerned with convergence rate and stopping criteria may need to be changed to delay convergence. If improvements in performance could be achieved with reasonable time and resource tradeoffs, such changes would certainly be desirable. The results of this simulation study are encouraging enough to warrant further investigation of this

$$
\text { matter. }
$$

It was disappointing that Hypergraph Clustering did not perform very well under most conditions, despite its intuitive appeal as a method that would find frequently-occurring multilocus genotypic patterns. The Hypergraph Clustering method has been reported to work well with very large variable sets (on the order of thousands), which have complex patterns for which large numbers of clusters
(10-20+) were relevant [43]. However, there has been no examination of the method's performance on smaller variable sets. Thus, it is possible that the restricted patterns present in our multilocus genotypic data were too simple and sparse and that the method is simply tuned to search for more complex patterns. Also, we were required to devise a translation of the resulting partitioning of genotypes into a clustering of individuals. We tested several such translations and implemented the best process out of several tested. Oftentimes, even when the method correctly chose the functional genotypes to be in different partitions, too many other nonfunctional genotypes were also chosen, which meant that the difference between an individual's likelihood of belonging to one cluster versus another was too small, making the choice of cluster assignment almost arbitrary. 


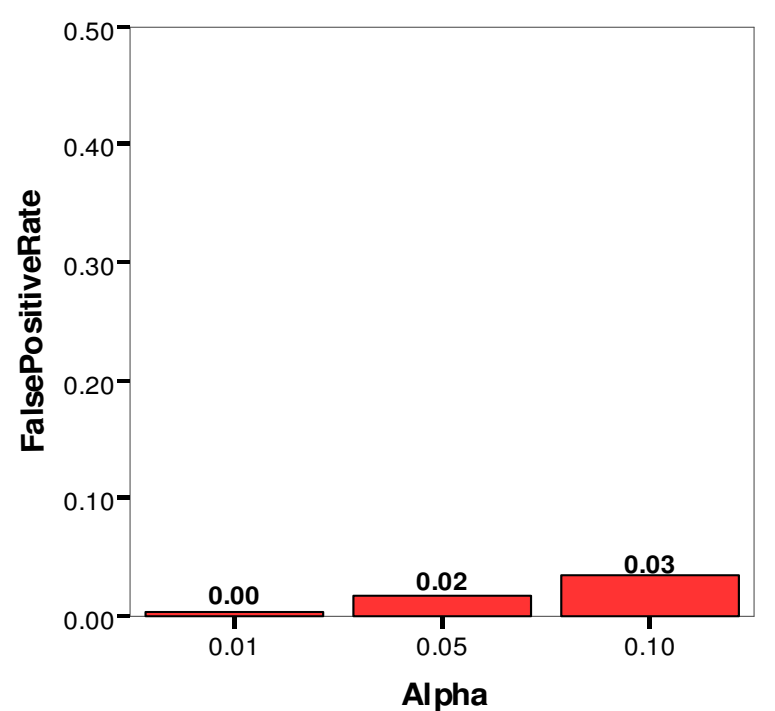

Figure 6

False Positive Rate by Significance Level (alpha)

The Fuzzy $k$-Modes Clustering method performed comparably to Bayesian Classification for the more complex datasets and was much less computationally intensive. It has been widely reported that the performance of $k$-means algorithms is highly variable depending on the method of seeding the initial cluster centroids [44]. While we used the recommended method of selecting individuals from the dataset to serve as the initial cluster modes, we perhaps could have achieved better results if we implemented an additional step to ensure that the initial centroids were substantially dissimilar to each other. This is supported by evidence that when the Fuzzy $k$-Modes Clustering resulted in only one cluster (effectively no partitioning of the data), the initial centroids were very similar and the method had converged early so that individuals had equal probability of belonging to any of the clusters. In such cases, the individual was arbitrarily assigned to the first cluster, thereby leading to all other clusters being empty.

As expected, the simpler the model, the better the performance of the three clustering algorithms, with the exception that the Hypergraph Clustering and Fuzzy $k$ Modes Clustering methods performed somewhat better on the THB (Trait Heterogeneity with Both locus heterogeneity and gene-gene interaction) datasets than they did on the THL (Trait Heterogeneity with Locus heterogeneity) and THG (Trait Heterogeneity with Gene-gene interaction) datasets. Likewise, in general, the fewer the nonfunctional loci and the larger the sample size, the better the performance was.

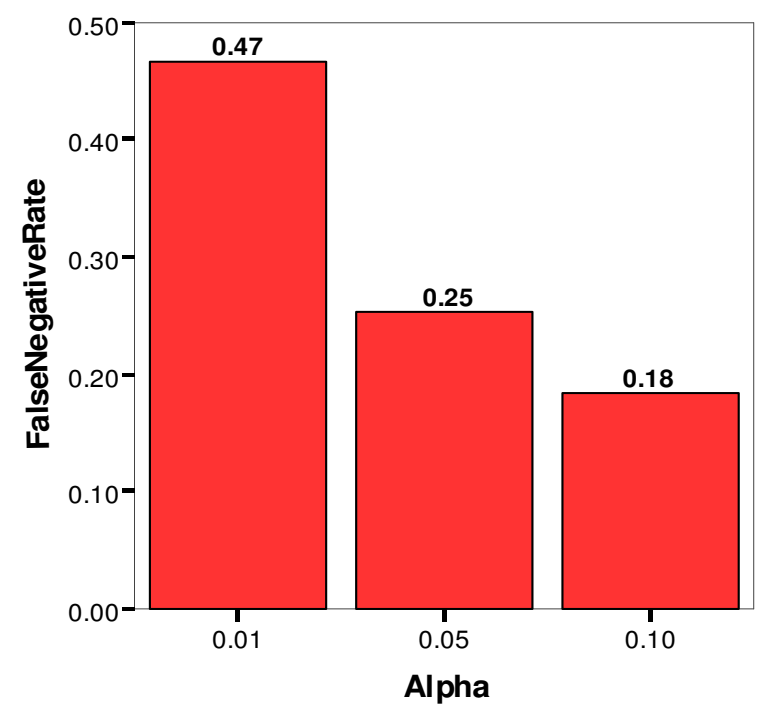

Figure 7

False Negative Rate by Significance Level (alpha)

\section{Applicability to real data}

To determine the efficacy of using the Bayesian Classification method on real data, the reliability of its internal clustering metrics at finding good clusterings was evaluated. Using the combination of the average log of class strength and the average cross class entropy to determine significance, the false positive rate was controlled very well, at three percent or less for all three significance levels. The false negative rate was acceptably low (18 percent) for the less stringent significance levels of 0.10 . However, it was high ( 47 percent) for the most stringent significance level of 0.01 . Thus, if a clustering of data were called significant according to permutation testing using either the average log of class strength or the average cross class entropy, we can be quite confident that the result were real. Typically geneticists prefer to accept a higher false positive rate to increase power; however, there is indeed a trade-off between these two types of error. Valuable time and resources can be spent on follow-up studies, and it can be very detrimental to pursue leads that do not have a good chance of yielding new information about the disease under study.

\section{Conclusion}

The efficacy of three clustering methodologies at uncovering trait heterogeneity in genotypic data was investigated. One method, Bayesian Classification, was found to perform very well under certain conditions (THO model) and to outperform the other methods. Permutation testing confirmed that the method could be used on real data with excellent Type I error control and acceptable Type II error control. By controlling the false positive rate so well, 

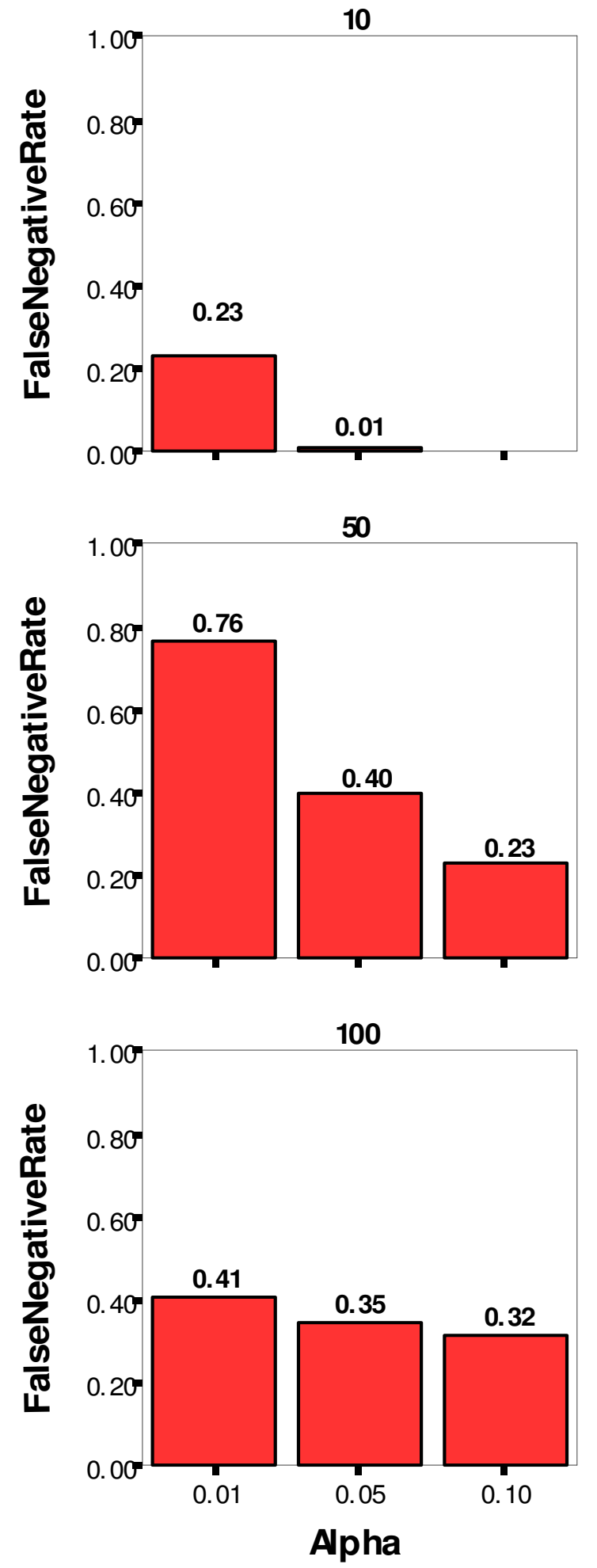

Figure 8

False Negative Rate by Significance Level (alpha) Paneled by Number of Nonfunctional Loci
Bayesian Classification offers a comfortable degree of certainty with regard to the hypotheses that it generates. This is true at least when the underlying data structure is similar to that simulated under the THO model. Further investigation of how different parameter settings may improve the performance of Bayesian Classification is planned.

\section{Methods \\ Data simulation}

To compare the performance of clustering methodologies in the task of uncovering trait heterogeneity in genotypic data, datasets were needed in which such heterogeneity was known to exist. Since there are no well-characterized real datasets available that fit this description, a simulation study was needed. Genetic models that contained two binary disease-associated traits, such that there is trait heterogeneity among 'affected' individuals, were used. In addition, some of the models incorporate locus heterogeneity, a gene-gene interaction, or both. Figure 2 depicts the structure of the four genetic models used to simulate the genotypic data.

Four prevalence levels were simulated for each genetic model: (1) fifteen percent, which is characteristic of a common disease phenotype such as obesity [45], (2) five percent, which is characteristic of a relatively common disease such as prostate cancer [46], (3) one percent, which is characteristic of a less common disease such as schizophrenia [47], and (4) one tenth of one percent, which is characteristic of a more uncommon disease such as multiple sclerosis [48]. Three realistic levels of sample size were simulated for each model: 200, 500 and 1000 affected individuals. Finally, four levels of non-functional loci were simulated: $0,10,50$ and 100 . The inclusion of non-functional loci adds a random noise effect that is present in real candidate gene studies in which the functional locus or loci are among many more suspected but actually non-functional loci. All loci, including the functional loci, were simulated to have equal biallelic frequencies of 0.5 .

Although the above parameter settings are by no means exhaustive of the biologically plausible situations, the outlined conditions are reasonable and specify 192 different sets of data specifications due to the combinatorial nature of the study design. To have adequate power to detect a difference in performance among clustering methodologies, 100 datasets per set of parameters, resulting in a total of 19,200 simulated data sets, were simulated.

For the purposes of simulating this data, a novel data simulation algorithm capable of incorporating these complex genetic factors in an epidemiologically-sound manner was designed and developed (Figure 10). Penetrance is the 

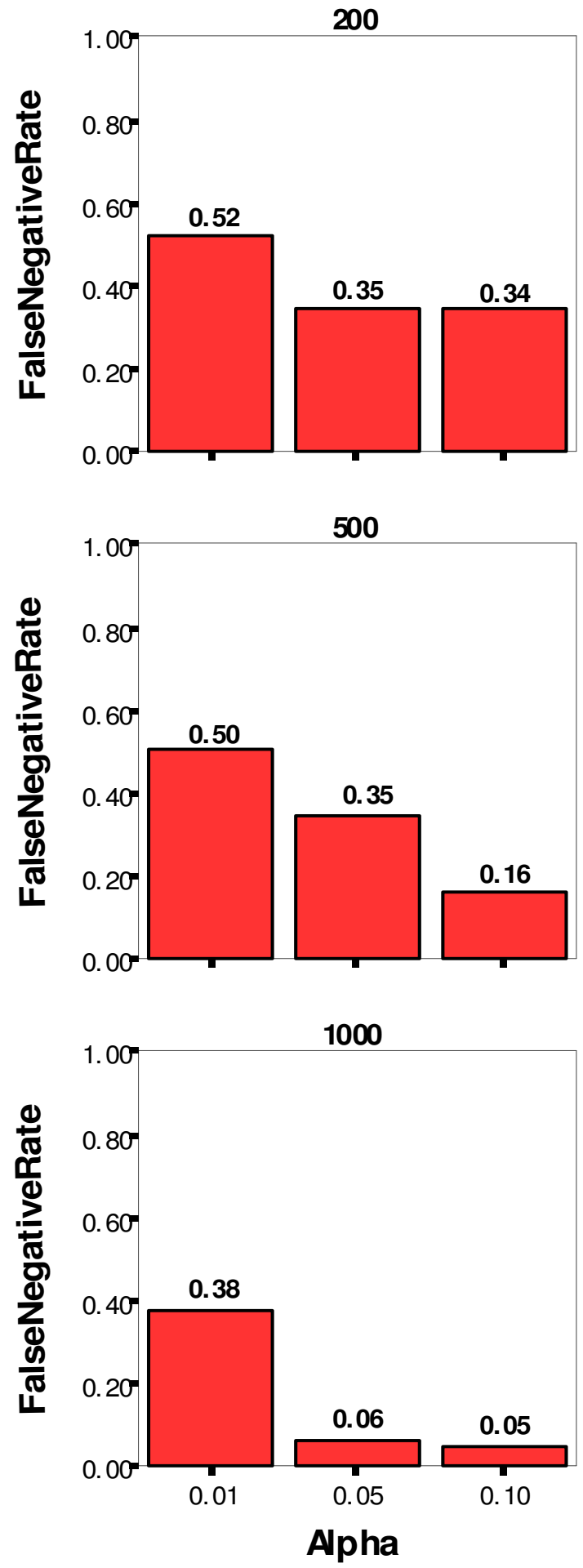

Figure 9

False Negative Rate by Significance Level (alpha) Paneled by Number of Affecteds probability of having a particular trait given a specific genotype (single or multilocus). Prevalence, on the other hand, is the percentage of individuals in a population that have a particular trait. The penetrance levels of the two simulated disease-associated traits are constrained by the overall prevalence level of the simulated disease. The two traits were simulated to contribute equally to the prevalence of the associated disease (fifty percent trait heterogeneity), such that a small but naturally occurring degree of overlap would be present, representing individuals having both disease-associated traits, instead of just one or the other. These penetrance tables are inputs for the new data simulation algorithm.

For one fourth of the models, trait heterogeneity only is involved (not locus heterogeneity or gene-gene interactions), and there is one genetic risk factor for each of the two traits. Each locus acts in a recessive manner, such that affected individuals have both copies of the high-risk allele at the disease-associated "functional" locus (Figure 11). A naturally occurring degree of overlap between the two traits can result, such that some affected individuals have the high-risk genotypes for both traits.

In the second quarter of the datasets, alocus heterogeneity described by Li and Reich [49] was also simulated (Figure 12(II)) so that for one of the traits, there are two associated loci, each of which is responsible for roughly half of the individuals affected with the trait. In that locus heterogeneity model, each of the functional loci acts in a recessive manner, such that the disease-associated genotype for the locus consists of two copies of the high-risk allele. For the other trait, a recessive model was implemented, as described above (Figure 12(I)). By chance, there might be some affected individuals who have the high-risk genotype from the first trait as well as one of the high-risk genotypes from the second trait.

In the third quarter of the datasets, a gene-gene interaction was simulated for one of the two traits. The "diagonal" gene-gene interaction model, first described by Frankel and Schork [50] and later by Li and Reich[49], which is nonlinear and nonadditive in nature, was used (Figure 13(II)). Under this model, a multilocus genotype is highrisk if it has exactly two high-risk alleles from either of the two associated loci. A multilocus genotype with fewer than or greater than two high-risk alleles is not associated with disease. For the other trait, a recessive model was implemented, as described above (Figure 13(I)). By chance, there might be some affected individuals who have the high-risk genotype from the first trait as well as one of the high-risk genotypes from the second trait.

In the fourth quarter of the datasets, one trait is simulated to involve locus heterogeneity (Figure 14(I)), while the 
Penetrance Function Array: each cell value represents the probability of having the diseaseassociated trait, given the (multilocus) genotype

Unaffecteds Probability Array: each cell value represents the probability of having the multilocus genotype given that the disease status is unaffected, which is the probability of being negative for all traits, or the joint probability of being negative for each trait, given the genotype frequency (prior probability)

Affecteds Probability Array: each cell value represents the probability of having the multilocus genotype given that the disease status is affected, which is the probability of being positive for at least one trait, which is the same as 1 - probability of being negative for all traits, or 1 - joint probability of being negative for each trait, given the genotype frequency (prior probability)

\section{Pseudocode:}

1. Allocate two probability arrays, one for Affecteds and one for Unaffecteds, each of size $\prod_{i}^{L} \sum_{j}^{A_{i}} j$ where $\mathrm{L}$ is the total number of loci and $\mathrm{A}_{\mathrm{i}}$ is the number of alleles for locus $\mathrm{i}$.

2. For each penetrance function $p$ (Status=Affected | Multilocus Genotype) $==>$ Distribute $1-p$ across relevant cells of Unaffecteds probability array

3. Populate cells of the Affecteds probability array with 1-(cell probability) of corresponding cells of the Unaffecteds probability array

4. For each locus $==>$ Distribute allele frequencies across appropriate cells of both probability arrays

5. Generate the specified number of unaffected individuals from the Unaffecteds probability array

6. Generate the specified number of affected individuals from the Affecteds probability array

7. Determine the status of each disease-associated trait for each affected individual thus.... If the affected individual has a high-risk genotype combination for that disease-associated trait, then that individual is affected for that trait. Otherwise, the individual is unaffected for that diseaseassociated trait. (By design, each affected individual will be affected at one or more diseaseassociated traits.)

\section{Figure 10}

Novel Data Simulation Algorithm. Simulates trait heterogeneity, locus heterogeneity and gene-gene interactions in an epidemiologically-sound manner. The inputs are penetrance function arrays, which are translated into probability arrays for affecteds and unaffecteds, separately. Then affected and unaffected individuals (with multilocus genotypes) are simulated from those respective arrays. 
(I)

\begin{tabular}{|c|c|c|}
\hline 1A1A & 1A1B & 1B1B \\
\hline 0 & 0 & $\mathrm{x}$ \\
\hline
\end{tabular}

(II)

\begin{tabular}{|c|c|c|}
\hline 2A2A & 2A2B & 2B2B \\
\hline 0 & 0 & $\mathrm{x}$ \\
\hline
\end{tabular}

\section{Figure I I}

Genetic Model THO (Trait Heterogeneity Only). The penetrance tables for Trait I and Trait II are presented. Cell values indicate penetrance level, or the probability of having the trait, given the corresponding multilocus genotype. For each of the two traits, a Mendelian recessive genetic model is used, in which the trait is penetrant only when two copies of the high risk (B) allele are present. The penetrance $(x)$ is constrained by the desired overall disease prevalence to be simulated $(0.00 \mathrm{I}, 0.0 \mathrm{I}, 0.05$ or 0.15$)$.

other is simulated to involve the "diagonal" gene-gene interaction, as described above (Figure 14(II)). Thus, there are some affected individuals who, by chance, will have one high-risk genotype from the first trait as well as one high-risk genotype from the second trait.

The input file for each of the clustering methods described below includes genotype and trait status information. Each row is a single individual. Column headings include unique individual number, trait status (affected for Trait 1, Trait 2, or both), and all simulated loci. Genotypes for each locus are encoded nominally (not ordinally), such that no genetic model assumptions are incorporated. Loci are numbered, and alleles are lettered. Thus, for a given locus ' 3 ' that has two alleles ' $\mathrm{A}$ ' and ' $\mathrm{B}$ ', the three possible genotypes are '3A3A', '3A3B', and '3B3B'. A different nomenclature could easily be used, however, since the methods simply treat each genotype as a character string for labeling purposes only and do not attribute any meaning or order to them.

\section{Clustering methods}

There exists a very large number of clustering algorithms and even more implementations of those algorithms. The choice of which clustering methodology to use should be determined by the kind of data being clustered and the purpose of the clustering [51]. Genotypic data is categorical, which immediately narrows the field of appropriate methods for this study to only a few. Three different clustering methodologies were chosen that are suitable for
(I)

\begin{tabular}{|c|c|c|}
\hline 1A1A & 1A1B & 1B1B \\
\hline 0 & 0 & $\mathrm{x}$ \\
\hline
\end{tabular}

(II)

\begin{tabular}{|c|c|c|c|}
\cline { 2 - 4 } \multicolumn{1}{c|}{} & 2A2A & 2A2B & 2B2B \\
\hline 3A3A & 0 & 0 & $x$ \\
\hline 3A3B & 0 & 0 & $x$ \\
\hline 3B3B & $\mathrm{x}$ & $\mathrm{x}$ & $\mathrm{x}$ \\
\hline
\end{tabular}

\section{Figure 12}

Genetic Model THL (Trait Heterogeneity with Locus Heterogeneity). The penetrance tables for Trait I and Trait II are presented. Cell values indicate penetrance level, or the probability of having the trait, given the corresponding multilocus genotype. For Trait I, a Mendelian recessive genetic model is used, in which the trait is penetrant only when two copies of the high risk (B) allele are present. For Trait II, a locus heterogeneity model described by Li and Reich [49] is used, in which the trait is penetrant only when two copies of the high risk allele at one or both loci are present (in this case the $B$ alleles for locus 2 and 3 are high risk).

categorical data and are appealing due to their speed or theoretical underpinnings.

The goal of this clustering is to find a partitioning of the affected individuals based on multilocus genotypic combinations that maps onto the trait heterogeneity simulated in the data. For example, consider a dataset with 10 loci (numbered 1 to 10), each of which has two alleles (A and $B)$, such that at each locus there are three possible genotypes ( $\mathrm{AA}, \mathrm{AB}$ and $\mathrm{BB})$. It is likely that among affected individuals in the dataset, subsets of individuals will share specific genotypes or multilocus combinations of genotypes (such as 2B2B; or 3A3B and 9A9B together), either by chance or because such combinations are related to genetic background, phenotypic variability, or trait heterogeneity in some way. Thus, a successful clustering would be one in which all the individuals who were simulated to have Trait I end up in one or more clusters that do not have any individuals unaffected for Trait I and all individuals who were simulated to have Trait II end up in one or more distinct clusters that do not have any individuals unaffected for Trait II (Figure 15). (Those individuals, who by chance have both Trait I and Trait II, could be 'correctly' placed in any cluster.) Such a clustering would effectively eliminate the noise present among affected individuals due to trait heterogeneity. In the case where 
(I)

\begin{tabular}{|c|c|c|}
\hline 1A1A & 1A1B & 1B1B \\
\hline 0 & 0 & $\mathrm{x}$ \\
\hline
\end{tabular}

(II)

\begin{tabular}{|c|c|c|c|}
\cline { 2 - 4 } \multicolumn{1}{c|}{} & $2 \mathrm{~A} 2 \mathrm{~A}$ & $2 \mathrm{~A} 2 \mathrm{~B}$ & $2 \mathrm{~B} 2 \mathrm{~B}$ \\
\hline $3 \mathrm{~A} 3 \mathrm{~A}$ & 0 & 0 & $\mathrm{x}$ \\
\hline $3 \mathrm{~A} 3 \mathrm{~B}$ & 0 & $0.5 \mathrm{x}$ & 0 \\
\hline $3 \mathrm{~B} 3 \mathrm{~B}$ & $\mathrm{x}$ & 0 & 0 \\
\hline
\end{tabular}

\section{Figure 13}

Genetic Model THG (Trait Heterogeneity with Gene-Gene Interaction). The penetrance tables for Trait I and Trait II are presented. Cell values indicate penetrance level, or the probability of having the trait, given the corresponding multilocus genotype. For Trait I, a Mendelian recessive genetic model is used, in which the trait is penetrant only when two copies of the high risk (B) allele are present. For Trait II, the "diagonal" genetic model first described by Frankel \& Schork [50] and later by Li and Reich [49] is used. Two loci (2 and 3 ) are involved, each with two alleles ( $A$ and $B)$, and the trait is penetrant only when exactly two copies of the high risk allele from either locus are present.

locus heterogeneity is also simulated, an even more successful clustering would be one in which there are two or more Trait II clusters, each of which has only those individuals who have a specific high-risk genotype (e.g., 2B2B from Figure 12) and none that do not.

The first clustering method is Bayesian Classification $[39,52]$. The corresponding AutoClass software is freely available from Peter Cheeseman at the NASA Ames Research Center. Bayesian Classification (BC) aims to find the most probable clustering of data given the data and the prior probabilities. In the case of genotypic data, prior probabilities are based on genotype frequencies, which for the purpose of the proposed data simulations are set in accordance with Hardy-Weinberg equilibrium and equal biallelic frequencies of 0.5 . The most probable clustering of data is determined from two posterior probabilities. The first involves the probability that a particular individual belongs to its 'assigned' cluster, or otherwise stated as the probability of the individual's multilocus genotype, conditional on it belonging to that cluster, with its characteristic genotypes. The second posterior probability involves the probability of a cluster given its assigned individuals, or otherwise stated as the probabil-
(I)

\begin{tabular}{|c|c|c|c|}
\cline { 2 - 4 } \multicolumn{1}{c|}{} & $1 \mathrm{~A} 1 \mathrm{~A}$ & $1 \mathrm{~A} 1 \mathrm{~B}$ & $1 \mathrm{~B} 1 \mathrm{~B}$ \\
\hline 2A2A & 0 & 0 & $\mathrm{x}$ \\
\hline 2A2B & 0 & 0 & $\mathrm{x}$ \\
\hline 2B2B & $\mathrm{x}$ & $\mathrm{x}$ & $\mathrm{x}$ \\
\hline
\end{tabular}

(II)

\begin{tabular}{|c|c|c|c|}
\cline { 2 - 4 } \multicolumn{1}{c|}{} & $3 \mathrm{~A} 3 \mathrm{~A}$ & $3 \mathrm{~A} 3 \mathrm{~A}$ & $3 \mathrm{~A} 3 \mathrm{~A}$ \\
\hline 4A4A & 0 & 0 & $\mathrm{x}$ \\
\hline 4A4B & 0 & $0.5 \mathrm{x}$ & 0 \\
\hline 4B4B & $\mathrm{x}$ & 0 & 0 \\
\hline
\end{tabular}

\section{Figure 14}

Genetic Model THB (Trait Heterogeneity with Both Locus Heterogeneity and Gene-Gene Interaction).

The penetrance tables for Trait I and Trait II are presented. Cell values indicate penetrance level, or the probability of having the trait, given the corresponding multilocus genotype. For Trait I, a locus heterogeneity model described by $\mathrm{Li}$ and Reich [49] is used, in which the trait is penetrant only when two copies of the high risk allele at one or both loci are present (in this case the B alleles for locus 2 and 3 are high risk). For Trait II, the "diagonal" genetic model first described by Frankel \& Schork [50] and later by Li and Reich [49] is used. Two loci (2 and 3 ) are involved, each with two alleles ( $A$ and $B)$, and the trait is penetrant only when exactly two copies of the high risk allele from either locus are present.

ity of the cluster's characteristic genotypes, conditional on the multilocus genotypes of the individuals assigned to that cluster.

In actuality, individuals are not 'assigned' to clusters in the hard classification sense but instead in the fuzzy sense they are temporarily assigned to the cluster to which they have the greatest probability of belonging. Thus, each individual has its own vector of probabilities of belonging to each of the clusters. The assignment of individuals is also not considered the most important result of the clustering method. A ranked listing is produced of all loci in the dataset with their corresponding normalized "attribute influence" values (ranging between 0 and 1), which provide a rough heuristic measure of relative influence of each locus in differentiating the classes from the overall data set. Thus, emphasis is placed on the identification of which attributes, or loci, are most important in producing the clustering. This information that can then 
(a)

\begin{tabular}{|c|c|c|c|c|c|c|c|c|c|c|c|c|}
\hline & \multicolumn{10}{|c|}{ Locus } \\
\hline Indiv & $\mathbf{1}$ & $\mathbf{2}$ & 3 & 4 & 5 & 6 & 7 & 8 & 9 & 10 & $\mathbf{1}$ & $\mathbf{2}$ \\
\hline 1 & $\mathrm{BB}$ & $\mathrm{AB}$ & $\mathrm{A}$ & $\mathrm{AB}$ & $\mathrm{AB}$ & $\mathrm{AA}$ & $\mathrm{AB}$ & $\mathrm{BB}$ & $\mathrm{AB}$ & $\mathrm{BB}$ & $\mathbf{X}$ & \\
\hline 2 & $\mathrm{AB}$ & $\mathrm{BB}$ & $\mathrm{BB}$ & $\mathrm{AB}$ & $\mathrm{BB}$ & $\mathrm{BB}$ & $\mathrm{AB}$ & $\mathrm{AB}$ & $\mathrm{BB}$ & $\mathrm{AB}$ & & $\mathbf{X}$ \\
\hline 3 & $\mathrm{BB}$ & $\mathrm{BB}$ & $\mathrm{AA}$ & $\mathrm{AA}$ & $\mathrm{AB}$ & $\mathrm{AB}$ & $\mathrm{AA}$ & $\mathrm{AB}$ & $\mathrm{BB}$ & $\mathrm{AB}$ & $\mathbf{X}$ & $\mathbf{X}$ \\
\hline 4 & $\mathrm{AB}$ & $\mathrm{BB}$ & $\mathrm{AB}$ & $\mathrm{AB}$ & $\mathrm{AB}$ & $\mathrm{AB}$ & $\mathrm{BB}$ & $\mathrm{AB}$ & $\mathrm{AA}$ & $\mathrm{AB}$ & & $\mathbf{X}$ \\
\hline 5 & $\mathrm{BB}$ & $\mathrm{AB}$ & $\mathrm{AA}$ & $\mathrm{AB}$ & $\mathrm{AA}$ & $\mathrm{AB}$ & $\mathrm{AA}$ & $\mathrm{AB}$ & $\mathrm{AA}$ & $\mathrm{BB}$ & $\mathbf{X}$ & \\
\hline 6 & $\mathrm{BB}$ & $\mathrm{AB}$ & $\mathrm{AB}$ & $\mathrm{AB}$ & $\mathrm{BB}$ & $\mathrm{BB}$ & $\mathrm{AB}$ & $\mathrm{AA}$ & $\mathrm{AB}$ & $\mathrm{AB}$ & $\mathbf{X}$ & \\
\hline 7 & $\mathrm{BB}$ & $\mathrm{BB}$ & $\mathrm{BB}$ & $\mathrm{BB}$ & $\mathrm{AB}$ & $\mathrm{AB}$ & $\mathrm{AA}$ & $\mathrm{AB}$ & $\mathrm{BB}$ & $\mathrm{AB}$ & $\mathbf{X}$ & $\mathbf{X}$ \\
\hline 8 & $\mathrm{AB}$ & $\mathrm{BB}$ & $\mathrm{AB}$ & $\mathrm{AB}$ & $\mathrm{AA}$ & $\mathrm{AA}$ & $\mathrm{AB}$ & $\mathrm{BB}$ & $\mathrm{AB}$ & $\mathrm{BB}$ & & $\mathbf{X}$ \\
\hline 9 & $\mathrm{BB}$ & $\mathrm{AA}$ & $\mathrm{AB}$ & $\mathrm{AB}$ & $\mathrm{BB}$ & $\mathrm{AB}$ & $\mathrm{AB}$ & $\mathrm{AA}$ & $\mathrm{AB}$ & $\mathrm{AB}$ & $\mathbf{X}$ & \\
\hline 10 & $\mathrm{AB}$ & $\mathrm{BB}$ & $\mathrm{AB}$ & $\mathrm{BB}$ & $\mathrm{AB}$ & $\mathrm{AB}$ & $\mathrm{BB}$ & $\mathrm{AB}$ & $\mathrm{AB}$ & $\mathrm{AA}$ & & $\mathbf{X}$ \\
\hline 11 & $\mathrm{AA}$ & $\mathrm{BB}$ & $\mathrm{AA}$ & $\mathrm{AA}$ & $\mathrm{AA}$ & $\mathrm{AB}$ & $\mathrm{AA}$ & $\mathrm{AB}$ & $\mathrm{AB}$ & $\mathrm{AB}$ & & $\mathbf{X}$ \\
\hline 12 & $\mathrm{BB}$ & $\mathrm{AB}$ & $\mathrm{BB}$ & $\mathrm{BB}$ & $\mathrm{AB}$ & $\mathrm{BB}$ & $\mathrm{AB}$ & $\mathrm{BB}$ & $\mathrm{AA}$ & $\mathrm{AB}$ & $\mathbf{X}$ & \\
\hline 13 & $\mathrm{AB}$ & $\mathrm{BB}$ & $\mathrm{AB}$ & $\mathrm{AA}$ & $\mathrm{AB}$ & $\mathrm{AB}$ & $\mathrm{BB}$ & $\mathrm{AB}$ & $\mathrm{AA}$ & $\mathrm{AA}$ & & $\mathbf{X}$ \\
\hline 14 & $\mathrm{BB}$ & $\mathrm{AA}$ & $\mathrm{AB}$ & $\mathrm{AB}$ & $\mathrm{BB}$ & $\mathrm{BB}$ & $\mathrm{AB}$ & $\mathrm{AA}$ & $\mathrm{AB}$ & $\mathrm{AB}$ & $\mathbf{X}$ & \\
\hline 15 & $\mathrm{AB}$ & $\mathrm{BB}$ & $\mathrm{BB}$ & $\mathrm{BB}$ & $\mathrm{AB}$ & $\mathrm{AA}$ & $\mathrm{AB}$ & $\mathrm{BB}$ & $\mathrm{AB}$ & $\mathrm{AA}$ & & $\mathbf{X}$ \\
\hline
\end{tabular}

(b)

\begin{tabular}{|c|c|c|c|c|c|c|c|c|c|c|c|c|}
\hline & \multicolumn{10}{|c|}{ Locus } \\
\hline Indiv & $\mathbf{1}$ & $\mathbf{2}$ & 3 & 4 & 5 & 6 & 7 & 8 & 9 & 10 & $\mathbf{1}$ & $\mathbf{2}$ \\
\hline 1 & $\mathrm{BB}$ & $\mathrm{AB}$ & $\mathrm{A}$ & $\mathrm{A}$ & $\mathrm{AB}$ & $\mathrm{AA}$ & $\mathrm{A}$ & $\mathrm{B}$ & $\mathrm{A}$ & $\mathrm{B}$ & $\mathbf{X}$ & \\
\hline 3 & $\mathrm{BB}$ & $\mathrm{BB}$ & $\mathrm{AA}$ & $\mathrm{AA}$ & $\mathrm{AB}$ & $\mathrm{AB}$ & $\mathrm{AA}$ & $\mathrm{AB}$ & $\mathrm{BB}$ & $\mathrm{AB}$ & $\mathbf{X}$ & $\mathbf{X}$ \\
\hline 5 & $\mathrm{BB}$ & $\mathrm{AB}$ & $\mathrm{AA}$ & $\mathrm{AB}$ & $\mathrm{AA}$ & $\mathrm{AB}$ & $\mathrm{AA}$ & $\mathrm{AB}$ & $\mathrm{AA}$ & $\mathrm{BB}$ & $\mathbf{X}$ & \\
\hline 6 & $\mathrm{BB}$ & $\mathrm{AB}$ & $\mathrm{AB}$ & $\mathrm{AB}$ & $\mathrm{BB}$ & $\mathrm{BB}$ & $\mathrm{AB}$ & $\mathrm{AA}$ & $\mathrm{AB}$ & $\mathrm{AB}$ & $\mathbf{X}$ & \\
\hline 9 & $\mathrm{BB}$ & $\mathrm{AA}$ & $\mathrm{AB}$ & $\mathrm{AB}$ & $\mathrm{BB}$ & $\mathrm{AB}$ & $\mathrm{AB}$ & $\mathrm{AA}$ & $\mathrm{AB}$ & $\mathrm{AB}$ & $\mathbf{X}$ & \\
\hline 12 & $\mathrm{BB}$ & $\mathrm{AB}$ & $\mathrm{BB}$ & $\mathrm{BB}$ & $\mathrm{AB}$ & $\mathrm{BB}$ & $\mathrm{AB}$ & $\mathrm{BB}$ & $\mathrm{AA}$ & $\mathrm{AB}$ & $\mathbf{X}$ & \\
\hline 14 & $\mathrm{BB}$ & $\mathrm{AA}$ & $\mathrm{AB}$ & $\mathrm{AB}$ & $\mathrm{BB}$ & $\mathrm{BB}$ & $\mathrm{AB}$ & $\mathrm{AA}$ & $\mathrm{AB}$ & $\mathrm{AB}$ & $\mathbf{X}$ & \\
\hline
\end{tabular}

(c)

\begin{tabular}{|c|c|c|c|c|c|c|c|c|c|c|c|c|}
\hline & \multicolumn{9}{|c|}{ Locus } & \multicolumn{2}{|c|}{ Trait } \\
\hline Indiv & $\mathbf{1}$ & $\mathbf{2}$ & 3 & 4 & 5 & 6 & 7 & 8 & 9 & 10 & $\mathbf{1}$ & $\mathbf{2}$ \\
\hline 2 & $\mathrm{AB}$ & $\mathrm{BB}$ & $\mathrm{BB}$ & $\mathrm{AB}$ & $\mathrm{BB}$ & $\mathrm{BB}$ & $\mathrm{AB}$ & $\mathrm{AB}$ & $\mathrm{BB}$ & $\mathrm{AB}$ & & $\mathbf{X}$ \\
\hline 4 & $\mathrm{AB}$ & $\mathrm{BB}$ & $\mathrm{AB}$ & $\mathrm{AB}$ & $\mathrm{AB}$ & $\mathrm{AB}$ & $\mathrm{BB}$ & $\mathrm{AB}$ & $\mathrm{AA}$ & $\mathrm{AB}$ & & $\mathbf{X}$ \\
\hline 7 & $\mathrm{BB}$ & $\mathrm{BB}$ & $\mathrm{BB}$ & $\mathrm{BB}$ & $\mathrm{AB}$ & $\mathrm{AB}$ & $\mathrm{AA}$ & $\mathrm{AB}$ & $\mathrm{BB}$ & $\mathrm{AB}$ & $\mathbf{X}$ & $\mathbf{X}$ \\
\hline 8 & $\mathrm{AB}$ & $\mathrm{BB}$ & $\mathrm{AB}$ & $\mathrm{AB}$ & $\mathrm{AA}$ & $\mathrm{AA}$ & $\mathrm{AB}$ & $\mathrm{BB}$ & $\mathrm{AB}$ & $\mathrm{BB}$ & & $\mathbf{X}$ \\
\hline 10 & $\mathrm{AB}$ & $\mathrm{BB}$ & $\mathrm{AB}$ & $\mathrm{BB}$ & $\mathrm{AB}$ & $\mathrm{AB}$ & $\mathrm{BB}$ & $\mathrm{AB}$ & $\mathrm{AB}$ & $\mathrm{AA}$ & & $\mathbf{X}$ \\
\hline 11 & $\mathrm{AA}$ & $\mathrm{BB}$ & $\mathrm{AA}$ & $\mathrm{AA}$ & $\mathrm{AA}$ & $\mathrm{AB}$ & $\mathrm{AA}$ & $\mathrm{AB}$ & $\mathrm{AB}$ & $\mathrm{AB}$ & & $\mathbf{X}$ \\
\hline 13 & $\mathrm{AB}$ & $\mathrm{BB}$ & $\mathrm{AB}$ & $\mathrm{AA}$ & $\mathrm{AB}$ & $\mathrm{AB}$ & $\mathrm{BB}$ & $\mathrm{AB}$ & $\mathrm{AA}$ & $\mathrm{AA}$ & & $\mathbf{X}$ \\
\hline 15 & $\mathrm{AB}$ & $\mathrm{BB}$ & $\mathrm{BB}$ & $\mathrm{BB}$ & $\mathrm{AB}$ & $\mathrm{AA}$ & $\mathrm{AB}$ & $\mathrm{BB}$ & $\mathrm{AB}$ & $\mathrm{AA}$ & & $\mathbf{X}$ \\
\hline
\end{tabular}

\section{Figure I 5}

Hypothetical Clustering of a THO Dataset. (a) A small dataset that consistent Trait Heterogeneity Only (THO) genetic model (see Figure II) is presented. All individuals with the high risk genotype (BB) at locus I have Trait I, and all individuals with the high risk genotype (BB) at locus 2 have Trait II. Some individuals have both high risk genotypes and, therefore, both traits. A successful clustering of this dataset might be one in which there are two clusters (b) and (c), such that one cluster contains only individuals who have Trait I (b) and the other cluster contains only individuals who have Trait II (c).

be used to more directly stratify affected (and/or unaffected) individuals, for instance, by using the top $n$ most influential loci identified, and to enable meaningful interpretation of the clustering result.

The second method is Hypergraph Clustering [40]. It has been implemented in the hMETIS software, which is freely available from George Karypis at the University of Minnesota. Hypergraph clustering seeks a partitioning of vertices, such that intracluster relatedness meets a specified threshold, while the weight of hyperedges cut by the par- titioning is minimized. In this case, vertices represent single locus genotypes, hyperedges represent association rules, and hyperedge weights represent the strength of the association rules. For instance, if a specific genotype at one locus co-occurs with a specific genotype at another locus, an association rule linking those two genotypes would be created, and that rule would have a weight equivalent to the proportion of individuals in the dataset that had both of those genotypes. Thus, for our purposes, association rules are multilocus genotype combinations that are found in the dataset. The freely available LPminer 
(a)

\begin{tabular}{|c|c|c|}
\hline Cluster 1 & Cluster 2 & Cluster 3 \\
\hline$\overline{1 B 1 B}$ & $\overline{2 B 2 B}$ & $\overline{7 \mathrm{~A} 7 \mathrm{~B}}$ \\
\hline $3 \mathrm{~A} 3 \mathrm{~B}$ & & 9A9A \\
\hline
\end{tabular}

(b)

\begin{tabular}{|c|c|c|c|c|c|c|c|c|c|c|c|c|c|}
\hline \multirow[b]{2}{*}{ Indiv } & \multicolumn{10}{|c|}{ Locus } & \multicolumn{3}{|c|}{$\begin{array}{l}\text { Percentage } \\
\text { of Matching } \\
\text { Genotypes } \\
\text { by Cluster }\end{array}$} \\
\hline & 1 & 2 & 3 & 4 & 5 & 6 & 7 & 8 & 9 & 10 & 1 & 2 & 3 \\
\hline 1 & BB & $A B$ & $A B$ & $A B$ & $\mathrm{AB}$ & AA & $A B$ & BB & $A B$ & BB & 100 & 0 & 50 \\
\hline 2 & $A B$ & BB & BB & $A B$ & $\mathrm{BB}$ & $\mathrm{BB}$ & $A B$ & $A B$ & BB & $A B$ & 0 & 100 & 50 \\
\hline 3 & BB & BB & AA & AA & $A B$ & $A B$ & AA & $A B$ & BB & $A B$ & 50 & 100 & 0 \\
\hline 4 & $A B$ & BB & $\mathrm{AB}$ & $\mathrm{AB}$ & $A B$ & $A B$ & BB & $\mathrm{AB}$ & AA & $\mathrm{AB}$ & 50 & 100 & 50 \\
\hline 5 & BB & $A B$ & AA & $\mathrm{AB}$ & AA & $\mathrm{AB}$ & AA & $\mathrm{AB}$ & AA & BB & $50^{*}$ & 0 & 50 \\
\hline 6 & BB & $\mathrm{AB}$ & $\mathrm{AB}$ & $\mathrm{AB}$ & BB & BB & $A B$ & AA & $\mathrm{AB}$ & $A B$ & 100 & 0 & 50 \\
\hline 7 & BB & BB & BB & BB & $\mathrm{AB}$ & $A B$ & AA & $A B$ & BB & $\mathrm{AB}$ & 50 & 100 & 0 \\
\hline 8 & $A B$ & BB & $A B$ & $\mathrm{AB}$ & AA & $\mathrm{AA}$ & $A B$ & BB & $\mathrm{AB}$ & BB & 50 & 100 & 50 \\
\hline 9 & BB & $\mathrm{AA}$ & $\mathrm{AB}$ & $\mathrm{AB}$ & $\mathrm{BB}$ & $A B$ & $A B$ & AA & $\mathrm{AB}$ & $A B$ & 100 & 0 & 50 \\
\hline 10 & $A B$ & BB & $\mathrm{AB}$ & BB & $A B$ & $A B$ & BB & $A B$ & $A B$ & AA & 50 & 100 & 0 \\
\hline 11 & AA & BB & AA & AA & AA & $A B$ & AA & $A B$ & $\mathrm{AB}$ & $\mathrm{AB}$ & 0 & 100 & 0 \\
\hline 12 & BB & $A B$ & BB & BB & $A B$ & BB & $A B$ & BB & $\mathrm{AA}$ & $A B$ & 50 & 0 & $100^{\star \star}$ \\
\hline 13 & $\mathrm{AB}$ & BB & $A B$ & AA & $A B$ & $A B$ & BB & $A B$ & AA & AA & 50 & 100 & 0 \\
\hline 14 & BB & AA & $\mathrm{AB}$ & $\mathrm{AB}$ & BB & BB & $A B$ & AA & $\mathrm{AB}$ & $\mathrm{AB}$ & 100 & 0 & 50 \\
\hline 15 & $\mathrm{AB}$ & BB & BB & BB & $\mathrm{AB}$ & AA & $A B$ & BB & $A B$ & AA & 0 & 100 & 50 \\
\hline
\end{tabular}

\section{Figure 16}

Example of Post-processing of Hypergraph Clustering Result. Hypergraph clustering produces a clustering of genotypes, instead of individuals. Thus, a clustering of individuals must be induced from this clustering of genotypes. As described in the text, an individual in assigned to the cluster for which it has the highest percentage of matching genotypes. Given the dataset presented in Figure I5(a) and a clustering of genotypes that is presented here (a), a clustering of individuals can be induced (b). For each individual (row), the percentage of matching genotypes that is highlighted indicates to which cluster the individual becomes assigned. Notice that for individual 5, there is a tie between the percentage of matching genotypes for clusters I and 3. In such cases, we arbitrarily assign the individual to the lower numbered cluster. Since cluster 3 does not contain any highrisk genotypes, it does not facilitate the goal of creating a clustering that maps to the simulated trait heterogeneity, and in the case of individual 12, it ends up capturing an individual who would preferably be clustered in cluster I.

program was used to generate the association rules [53]. LPminer searches the database for multilocus genotype combinations that appear together with substantial frequency (above a prespecified "support" percentage) and outputs this info as a list of association rules. hMETIS takes these association rules and uses them to create a hypergraph in which single locus genotypes are vertices and association rules dictate the presence and weight of hyperedges. hMETIS creates a partition of the hypergraph such that the weight of the removed hyperedges is minimized. It achieves this by using a series of phases, somewhat analogous to the stages of a simulated annealing algorithm, in an attempt to avoid making decisions which are only locally (not globally) optimal.

This process results in a partitioning (or clustering) of the genotypes in a dataset. If a single dataset was being analyzed, this information by itself could be sufficiently helpful since it would provide information about which multilocus genotypes appear with such frequency that they characterize groups of individuals. Individuals could be directly stratified using such multilocus combinations (similar to the way attribute influence values in the Bayesian Classification method could be used). However, for the purpose of comparing the results of Hypergraph Partitioning to those of the other two methods, which produce clusters, or partitions, of individuals (not genotypes), such a partitioning of individuals still needed to be created. Since a given individual could have more than one of the multilocus genotypes specified by different hyperedges in the final partitioning, the partitioning of individuals was not entirely straightforward. Thus, a heuristic was devised such that each individual would be assigned to the partition, or cluster, for which it had the highest percentage of matching genotypes (Figure 16). More specifically, for each cluster, the number of loci represented by one or more genotypes in that cluster was determined $\left(\mathrm{L}_{\mathrm{c}}\right)$. Then, for each individual, for each cluster, the 
(a)

\begin{tabular}{|c|c|c|c|c|c|c|c|c|c|c|}
\hline & \multicolumn{10}{|c|}{ Locus } \\
\hline Cluster & 1 & 2 & 3 & 4 & 5 & 6 & 7 & 8 & 9 & 10 \\
\hline $1(1)$ & BB & $A B$ & $A B$ & $A B$ & $A B$ & $\mathrm{AA}$ & $A B$ & BB & $A B$ & BB \\
\hline $2(5)$ & $\mathrm{BB}$ & $A B$ & $\overline{A A}$ & $\mathrm{AB}$ & AA & $\mathrm{AB}$ & AA & $\mathrm{AB}$ & AA & $\mathrm{BB}$ \\
\hline $3(12)$ & BB & $A B$ & BB & BB & $A B$ & BB & $A B$ & BB & AA & $A B$ \\
\hline $4(15)$ & $A B$ & BB & BB & BB & $A B$ & AA & $A B$ & BB & $A B$ & AA \\
\hline
\end{tabular}

\begin{tabular}{|c|c|c|c|c|c|c|c|c|c|c|c|c|c|c|}
\hline \multirow[b]{2}{*}{ Indiv } & \multicolumn{10}{|c|}{ Locus } & \multicolumn{4}{|c|}{$\begin{array}{c}\text { Cluster } \\
\text { Distance }\end{array}$} \\
\hline & 1 & 2 & 3 & 4 & 5 & 6 & 7 & 8 & 9 & 10 & 1 & 2 & 3 & 4 \\
\hline 1 & BB & $A B$ & $A B$ & $A B$ & $A B$ & AA & $A B$ & BB & $A B$ & BB & 0 & 6 & 5 & 5 \\
\hline 2 & $\mathrm{AB}$ & BB & BB & $A B$ & BB & $\mathrm{BB}$ & $\mathrm{AB}$ & $A B$ & BB & $\mathrm{AB}$ & 8 & 8 & 6 & 6 \\
\hline 3 & BB & BB & AA & AA & $A B$ & $A B$ & AA & $A B$ & BB & $A B$ & 8 & 5 & 7 & 8 \\
\hline 4 & $\mathrm{AB}$ & BB & $A B$ & $A B$ & $A B$ & $A B$ & BB & $A B$ & AA & $\mathrm{AB}$ & 7 & 6 & 7 & 7 \\
\hline 5 & BB & $A B$ & AA & $A B$ & $\overline{A A}$ & $\overline{A B}$ & $\overline{A A}$ & $A B$ & AA & BB & 6 & 0 & 5 & 10 \\
\hline 6 & BB & $A B$ & $A B$ & $A B$ & BB & BB & $A B$ & AA & $A B$ & $A B$ & 4 & 7 & 5 & 8 \\
\hline 7 & BB & BB & BB & BB & $A B$ & $A B$ & AA & $A B$ & BB & $\mathrm{AB}$ & 8 & 6 & 5 & 6 \\
\hline 8 & $\mathrm{AB}$ & BB & $A B$ & $\mathrm{AB}$ & AA & AA & $A B$ & BB & $\mathrm{AB}$ & $\mathrm{BB}$ & 4 & 7 & 8 & 4 \\
\hline 9 & BB & AA & $A B$ & $A B$ & BB & $A B$ & $A B$ & AA & $A B$ & $\mathrm{AB}$ & 5 & 7 & 8 & 8 \\
\hline 10 & $A B$ & BB & $A B$ & BB & $A B$ & $A B$ & BB & $A B$ & $A B$ & AA & 7 & 8 & 8 & 4 \\
\hline 11 & AA & BB & AA & AA & AA & $A B$ & AA & $A B$ & $A B$ & $\mathrm{AB}$ & 9 & 5 & 9 & 8 \\
\hline 12 & BB & $A B$ & BB & BB & $A B$ & BB & $A B$ & BB & AA & AB & 5 & 7 & 0 & 5 \\
\hline 13 & $A B$ & BB & $A B$ & AA & $A B$ & $A B$ & BB & $A B$ & AA & $\mathrm{AA}$ & 8 & 7 & 8 & 6 \\
\hline 14 & BB & AA & $A B$ & $A B$ & BB & BB & $A B$ & AA & $A B$ & $\mathrm{AB}$ & 5 & 7 & 6 & 8 \\
\hline 15 & $A B$ & BB & BB & BB & $A B$ & $\mathrm{AA}$ & $A B$ & BB & $A B$ & AA & 5 & 10 & 5 & 0 \\
\hline
\end{tabular}

(c)

\begin{tabular}{|l|c|c|c|c|c|c|c|c|c|c|}
\hline & \multicolumn{10}{|c|}{ Locus } \\
\hline Cluster & $\mathbf{1}$ & $\mathbf{2}$ & $\mathbf{3}$ & $\mathbf{4}$ & $\mathbf{5}$ & $\mathbf{6}$ & $\mathbf{7}$ & $\mathbf{8}$ & $\mathbf{9}$ & $\mathbf{1 0}$ \\
\hline $\mathbf{1}$ & $\mathrm{BB}$ & $\mathrm{AA}$ & $\mathrm{AB}$ & $\mathrm{AB}$ & $\mathrm{BB}$ & $\mathrm{AA}$ & $\mathrm{AB}$ & $\mathrm{AA}$ & $\mathrm{AB}$ & $\mathbf{A B}$ \\
\hline $\mathbf{2}$ & $\mathrm{BB}$ & $\mathrm{BB}$ & $\mathrm{AA}$ & $\mathrm{AA}$ & $\mathrm{AA}$ & $\mathrm{AB}$ & $\mathrm{AA}$ & $\mathrm{AB}$ & $\mathrm{AA}$ & $\mathrm{AB}$ \\
\hline $\mathbf{3}$ & $\mathrm{BB}$ & $\mathrm{BB}$ & $\mathrm{BB}$ & $\mathrm{BB}$ & $\mathrm{AB}$ & $\mathrm{BB}$ & $\mathrm{AB}$ & $\mathrm{AB}$ & $\mathrm{BB}$ & $\mathrm{AB}$ \\
\hline $\mathbf{4}$ & $\mathrm{AB}$ & $\mathrm{BB}$ & $\mathrm{AB}$ & $\mathrm{BB}$ & $\mathrm{AB}$ & $\mathrm{AB}$ & $\mathrm{BB}$ & $\mathrm{AB}$ & $\mathrm{AB}$ & $\mathrm{AA}$ \\
\hline
\end{tabular}

\begin{tabular}{|c|c|c|c|c|c|c|c|c|c|c|c|c|c|c|}
\hline & \multicolumn{10}{|c|}{ Locus } & \multicolumn{4}{|c|}{$\begin{array}{c}\text { Cluster } \\
\text { Distance }\end{array}$} \\
\hline Indiv & 1 & 2 & 3 & 4 & 5 & 6 & 7 & 8 & 9 & 10 & 1 & 2 & 3 & 4 \\
\hline 1 & BB & $A B$ & $A B$ & $A B$ & $A B$ & $\mathrm{AA}$ & $A B$ & BB & $\mathrm{AB}$ & BB & 4 & 9 & 7 & 7 \\
\hline 2 & $A B$ & BB & BB & $A B$ & BB & BB & $A B$ & $A B$ & BB & $\mathrm{AB}$ & 6 & 7 & 3 & 7 \\
\hline 3 & $\mathrm{BB}$ & BB & AA & $\mathrm{AA}$ & $\mathrm{AB}$ & $\mathrm{AB}$ & AA & $A B$ & BB & $\mathrm{AB}$ & 8 & 3 & 4 & 6 \\
\hline 4 & $A B$ & BB & $A B$ & $A B$ & $A B$ & $\mathrm{AB}$ & BB & $A B$ & AA & $\mathrm{AB}$ & 7 & 6 & 6 & 3 \\
\hline 5 & BB & $A B$ & AA & $A B$ & $\mathrm{AA}$ & $\mathrm{AB}$ & AA & $A B$ & AA & BB & 8 & 3 & 8 & 8 \\
\hline 6 & $\mathrm{BB}$ & $A B$ & $\mathrm{AB}$ & $A B$ & BB & $\mathrm{BB}$ & $A B$ & AA & $A B$ & $\mathrm{AB}$ & 2 & 8 & 6 & 8 \\
\hline 7 & BB & BB & BB & BB & $A B$ & $A B$ & AA & $A B$ & BB & $\mathrm{AB}$ & 8 & 4 & 2 & 5 \\
\hline 8 & $A B$ & BB & $A B$ & $A B$ & AA & $\mathrm{AA}$ & $A B$ & BB & $A B$ & BB & 5 & 8 & 8 & 6 \\
\hline 9 & BB & AA & $A B$ & $A B$ & BB & $A B$ & $A B$ & AA & $A B$ & $A B$ & 1 & 7 & 7 & 7 \\
\hline 10 & $A B$ & BB & $A B$ & BB & $A B$ & $\mathrm{AB}$ & BB & $A B$ & $A B$ & AA & 8 & 7 & 6 & 0 \\
\hline 11 & AA & BB & AA & AA & AA & $\mathrm{AB}$ & AA & $A B$ & $A B$ & $A B$ & 8 & 2 & 7 & 6 \\
\hline 12 & BB & $A B$ & BB & BB & $A B$ & BB & $A B$ & BB & AA & $\mathrm{AB}$ & 7 & 7 & 3 & 8 \\
\hline 13 & $A B$ & BB & $A B$ & AA & $A B$ & $A B$ & BB & $A B$ & AA & AA & 9 & 5 & 7 & 2 \\
\hline 14 & BB & AA & $\mathrm{AB}$ & $A B$ & BB & BB & $A B$ & AA & $A B$ & $A B$ & 1 & 8 & 6 & 8 \\
\hline 15 & $A B$ & BB & BB & BB & $A B$ & AA & $A B$ & BB & $A B$ & AA & 7 & 9 & 5 & 4 \\
\hline
\end{tabular}

\section{Figure 17}

Example of $\boldsymbol{k}$-Modes Clustering. In this example, the same dataset presented in Figure 15 is used to demonstrate the different steps involved the $k$-modes clustering algorithm. In this example, $k$ was chosen to be 4 , such that four clusters will initially be formed. (a) The cluster centroids are seeded by randomly selecting the genotypes of actual individuals in the dataset. The number in parentheses beside the cluster number is the individual used to seed that cluster. (b) Individuals are then compared to each of the cluster centroids, and the number of nonmatching genotypes between each cluster centroid and that individual are recorded. The individual is then assigned to the cluster for which it had the fewest number of nonmatching genotypes (in bold). (c) The next step is to update the cluster centroids based on the individuals now assigned to the clusters. The mode genotype among individuals assigned to a cluster becomes the centroid genotype at that locus. Genotypes that changed from the initialization to the update are shown in bold. (d) After the centroids are updated, the individuals are reevaluated as to which cluster they most closely resemble and are assigned to that cluster. Only cluster 4 was assigned to a different cluster than it was previously. Steps (c) and (d) are repeated until no genotypes are changed in any cluster centroid and no individuals' cluster assignments are changed. 
number of matching genotypes between the cluster and the individual $\left(\mathrm{M}_{\mathrm{ic}}\right)$ was divided by $\mathrm{L}_{\mathrm{C}^{\prime}}$ producing a vector of similarity percentages per individual, similar to the vector of probabilities used by the Bayesian Classification and Fuzzy $k$-Modes Clustering methods. Each individual was then assigned to the cluster with which it had the greatest similarity.

The third clustering method is Fuzzy $k$-Modes Clustering [41]. $k$-Modes is a trivial extension of the popular $k$-means algorithm to categorical data. In both methods, cluster centroids can be initialized at random or by one of many seeding strategies [44], and individuals are assigned to their nearest cluster centroids. Then, cluster centroids are reevaluated based on their newly assigned individuals. For the $k$-means algorithm, the centroid is calculated as the mean vector of genotypes across individuals. However, for nominal data, such means are not necessarily meaningful, and the $k$-modes algorithm instead determines the centroid as the mode vector of genotypes across individuals. Genotypes are encoded nominally (not ordinally), such that no two genotypes are considered 'closer' than another two, and the 'distance' between an individual and a centroid is calculated as the cumulative number of nonmatching genotypes across all loci. After cluster centroids are reevaluated, individuals are again assigned to their nearest centroids, and this process is repeated until the assignment of individuals to clusters does not change. Figure 17 demonstrates the first steps of the $k$-modes clustering, using the same dataset presented in Figures 15 and 16. The straightforward algorithm was developed in the $\mathrm{C}++$ language. The number of clusters $(k)$ was prespecified to be $2,3,4,5$ or 6 . All five possible $k$ were run for each dataset. Each cluster centroid was initially set to the values of a randomly selected individual in the dataset being analyzed. Both a 'fuzzy' and a 'hard' version of the $k$ modes algorithm were implemented and tested, and while their results on test datasets were comparable, the fuzzy version did perform slightly better and provided more information, which could be used for interpretation of results. Thus, the fuzzy version was chosen for use in these analyses.

\section{Statistical analysis}

\section{Comparison of clustering methods}

Each clustering method has its own metric(s) for evaluating the "goodness" of a clustering of data. Since these methods are being tested on simulated data, classification error of a given clustering can be calculated as the number of misclassified individuals divided by the total number of individuals. However, simple classification error has its disadvantages. Firstly, in cases such as this where there is overlap between the known classes, the researcher must make an arbitrary decision as to when individuals who have been simulated to have both traits, not just one or the other, are considered to be misclassified. The decision about error is equally arbitrary when the number of resulting clusters is greater than the number of known classes. For instance, if the individuals belonging to one class were divided into two classes by the clustering algorithm, calculating classification error would require either (1) that none of those individuals be considered incorrectly classified, since they are all in homogenous clusters, or else (2) that all individuals from one of those clusters be considered misclassified. Neither choice seems to satisfactorily capture the "goodness" of the clustering result. Subsequently, it is not advisable to compare the classification error of two clustering results for which the number of clusters differs.

It is for these reasons alternative cluster recovery metrics were investigated. The Hubert-Arabie Adjusted Rand Index $\left(\mathrm{ARI}_{\mathrm{HA}}\right)$ addresses the concerns raised by classification error and was, therefore, chosen to evaluate the goodness of clustering results from the three clustering methods being compared [42]. Calculation of the ARI ${ }_{\mathrm{HA}}$ involves determining (1) whether pairs of individuals, who were simulated to have the same trait, are clustered together or apart and (2) whether pairs of individuals, who do not have the same trait, are clustered together or apart. The $\mathrm{ARI}_{\mathrm{HA}}$ is robust with regard to the number of individuals to cluster, the number of resulting clusters, and the relative size of those clusters [54]. It is, however, sensitive to the degree of class overlap, which is desirable since it will penalize more for good clusterings that occur by chance than classification error would. When interpreting $\mathrm{ARI}_{\mathrm{HA}}$ values, 0.90 and greater can be considered excellent recovery, 0.80 and greater is good recovery, 0.65 and greater reflects moderate recovery, and less than 0.65 indicates poor recovery. These values were derived from empirical studies showing observations cut at the 95th, 90th, 85th and 80th percentiles corresponded to ARI $\mathrm{HA}_{\mathrm{HA}}$ values of $0.86,0.77,0.67$ and 0.60 respectively [54].

The $\mathrm{ARI}_{\mathrm{HA}}$ was used as the gold standard measure to compare the performance of the three clustering methods. Three categorical variables were created that could be tested using the nonparametric chi-square test of independence. The $\mathrm{ARI}_{\mathrm{HA}}$ values were discretized into a 1 or 0 depending on whether they met or exceeded the cutoff values for excellent, good and moderate cluster recovery, as described above. A chi-square test of independence was performed testing the null hypothesis that the number of clusterings achieving a certain $\mathrm{ARI}_{\mathrm{HA}}$ value was independent of the clustering method. Five percent was chosen as the significance level (alpha). An evaluation was performed of whether one method significantly outperformed the others and whether that method performed satisfactorily accordingly to the ARI $\mathrm{HA}_{\mathrm{HA}}$. 


\section{Applicability to real data}

As a reminder, the ultimate goal of this research is to find a clustering method that works well at uncovering trait heterogeneity in real genotypic data. Unlike for the current simulation study, for real data to which clusters individuals belong is not known a priori, otherwise the clustering would not be necessary. Indeed, it is the goal of clustering to uncover natural clusters or partitions of data using the method-specific "goodness" metric as a guide. In preparation for application of a clustering method to real data, after choosing the superior method, that method's internal clustering metrics were analyzed using permutation testing to determine how good a proxy they are for $\mathrm{ARI}_{\mathrm{HA}}$.

The Bayesian Classification method produces two internal clustering metrics for each resulting cluster, or class: (1) class strength, and (2) cross-class entropy. Class strength is a heuristic measure of how strongly each class predicts "its" instances and is reported as the log of class strength. Cross-class entropy is a measure of how strongly the class probability distribution function differs from that of the dataset as a whole. Because each metric is reported per resulting cluster, or class, the average metric value across clusters was calculated and utilized for evaluating cluster fitness.

The ratio of one hundred permuted datasets per simulated dataset was chosen, which should result in a reasonable approximation of the null distribution but would not put unreasonable strain on resources and time [55]. Genotypes were permuted within loci across individuals, such that the overall frequency of genotypes at any one locus was unchanged, but the frequency of multilocus genotypes was altered at random. This created a null sample in which the frequency of multilocus genotypes was no longer associated with trait status except by chance. The empirically-determined superior clustering method was applied to each permuted data set and both the internal clustering metric values and the $\mathrm{ARI}_{\mathrm{HA}}$ were determined. For each set of 100 permuted data sets, the significance of each of the simulated dataset results was determined based on whether they exceeded the values at the significance level in the corresponding null distribution. Ten percent was chosen as the acceptable Type I error rate since these methods serve as a means of data exploration to be followed by more rigorous, supervised analyses on individual clusters of the data. However, the more conventional levels of 0.05 and 0.01 were also evaluated.

Finally, the ability of permutation testing to preserve an acceptable Type I (false positive) error rate was evaluated at the three specified significance levels. A false positive was defined as a clustering result which had a p-value according to at either of the internal clustering metrics that was significant but had a p-value according to $\mathrm{ARI}_{\mathrm{HA}}$ that was not significant. The Type II (false negative) error rate was evaluated at the same alpha levels to determine the sensitivity for detecting trait heterogeneity when it is present. A false negative was defined as a clustering result which had a p-value that was not significant according to both of the internal clustering metrics but had a p-value that was significant according to $\mathrm{ARI}_{\mathrm{HA}}$.

\section{Abbrevations \\ $\mathrm{ARI}_{\mathrm{HA}}$ Hubert-Arabie Adjusted Rand Index}

THO Trait Heterogeneity Only

THL Trait Heterogeneity with Locus Heterogeneity

\section{THG Trait Heterogeneity with Gene-Gene Interaction}

THB Trait Heterogeneity with Both Locus Heterogeneity and Gene-Gene Interaction

\section{Authors' contributions}

TATW conceived of the study, performed all data simulations, implemented the three clustering methods, conducted the comparative data analysis, and drafted the manuscript. JHM and JLH participated in the design of the study and helped to draft the background section of the manuscript. All authors read and approved the final manuscript.

\section{Acknowledgements}

This work has been funded by NLM training grant T32 MH649/ 3 and by NIH grants HL65234, AI59694, N532830, A6I9085, HD047447 and RR018787. The authors would like to acknowledge the contribution of Scott M. Dudek, who programmed the Fuzzy k-Modes Clustering algorithm. He was funded by NIH grant HL065962. The authors would also like to thank Marylyn Ritchie, Lance Hahn and Bill White for their thoughtful input on study design.

\section{References}

I. Thornton-Wells TA, Moore JH, Haines JL: Genetics, statistics and human disease: analytical retooling for complexity. Trends Genet 2004, 20:640-647.

2. Rivolta C, Sharon D, DeAngelis MM, Dryja TP: Retinitis pigmentosa and allied diseases: numerous diseases, genes, and inheritance patterns. Hum Mol Genet 2002, I I:1219-1227.

3. Kulczycki LL, Kostuch M, Bellanti JA: A clinical perspective of cystic fibrosis and new genetic findings: relationship of CFTR mutations to genotype-phenotype manifestations. Am J Hum Genet 2003, I I6A:262-267.

4. Povey S, Burley MW, Attwood J, Benham F, Hunt D, Jeremiah SJ, Franklin D, Gillett G, Malas S, Robson EB, Tippett P, Edwards JH, Kwiatkowski DJ, Super M, Mueller R, Fryer A, Clarke A, Webb D, Osborne J: Two loci for tuberous sclerosis: one on $9 q 34$ and one on 16p I3. Ann Hum Genet 1994, 58: 107-I27.

5. Young J, Povey S: The genetic basis of tuberous sclerosis. Mol Med Today 1998, 4:313-319.

6. Harding AE: The clinical features and classification of the late onset autosomal dominant cerebellar ataxias: a study of I I families, including descendants of 'the Drew family of Walworth.'. Brain 1982, 105: 1-28. 
7. Rosenberg RN: Autosomal dominant cerebellar phenotypes: the genotype has settled the issue. Neurology 1995, 45:1-5.

8. Devos D, Schraen-Maschke S, Vuillaume I, Dujardin K, Naze P, Willoteaux C, Destee A, Sablonniere B: Clinical features and genetic analysis of a new form of spinocerebellar ataxia. Neurology 200I, 56:234-238.

9. Tager-Flusberg $\mathrm{H}$, Joseph RM: Identifying neurocognitive phenotypes in autism. Philos Trans R Soc Lond B Biol Sci 2003, 358:303-3I4.

10. Bradford Y, Haines JL, Hutcheson H, Gardiner M, Braun T, Sheffield V, Cassavant T, Huang W, Wang K, Vieland V, Folstein S, Santangelo $S$, Piven J: Incorporating language phenotypes strengthens evidence of linkage to autism. Am J Med Genet 200I, 105:539-547.

11. Shao Y, Raiford KL, Wolpert CM, Cope HA, Ravan SA, Ashley-Koch AA, Abramson RK, Wright HH, DeLong RG, Gilbert JR, Cuccaro ML, Pericak-Vance MA: Phenotypic homogeneity provides increased support for linkage on chromosome 2 in autistic disorder. Am J Hum Genet 2002, 70: |058-I06I.

12. Carrasquillo MM, McCallion AS, Puffenberger EG, Kashuk CS, Nouri $\mathrm{N}$, Chakravarti A: Genome-wide association study and mouse model identify interaction between RET and EDNRB pathways in Hirschsprung disease. Nat Genet 2002, 32:237-244.

13. Doh-ura K, Tateishi J, Sasaki H, Kitamoto T, Sakaki Y: Pro-to-leu change at position 102 of prion protein is the most common but not the sole mutation related to Gerstmann-Straussler syndrome. Biochem Biophys Res Comm 1989, 163:974-979.

14. Owen F, Poulter M, Collinge J, Crow TJ: A codon I29 polymorphism in the PRIP gene. Nucleic Acids Res 1990, 18:3103.

15. Collinge J, Palmer MS, Dryden AJ: Genetic predisposition to iatrogenic Creutzfeldt-Jakob disease. Lancet I99I, 337:144I-1442.

16. Palmer MS, Dryden AJ, Hughes JT, Collinge J: Homozygous prion protein genotype predisposes to sporadic Creutzfeldt-Jakob disease. Nature 1991, 352:340-342.

17. De Silva R, Ironside JW, McCardle L, Esmonde T, Bell J, Will R, Windl O, Dempster M, Estibeiro P, Lathe R: Neuropathological phenotype and 'prion protein' genotype correlation in sporadic Creutzfeldt-Jakob disease. Neurosci Lett 1994, 179:50-52.

18. Doh-ura K, Kitamoto T, Sakaki Y, Tateishi J: CJD discrepancy. Nature 1991, 353:80I-802.

19. Goldfarb LG, Brown P, Haltia M, Cathala F, McCombie WR, Kovanen J, Cervenakova L, Goldin L, Nieto A, Godec MS, Asher DM, Gajdusek DC: Creutzfeldt-Jakob disease cosegregates with the codon I 78Asn PRNP mutation in families of European origin. Ann Neurol 1992, 3 I:274-28I.

20. Hirschhorn JN, Lohmueller K, Byrne E, Hirschhorn K: A comprehensive review of genetic association studies. Genet Med 2002, 4:45-6I.

21 . Ott J: Strategies for characterizing highly polymorphic markers in human gene mapping. Am J Hum Genet 1992, 5 I:283-290.

22. Smith $C A B$ : Testing for heterogeneity of recombination fraction values in human genetics. Annals of Human Genetics 1963 27:175-182.

23. Ritchie MD, Hahn LW, Roodi N, Bailey LR, Dupont WD, Parl FF, Moore $\mathrm{JH}$ : Multifactor-dimensionality reduction reveals highorder interactions among estrogen-metabolism genes in sporadic breast cancer. Am J Hum Genet 200I, 69:I38-147.

24. Ritchie MD, Hahn LW, Moore JH: Power of multifactor dimensionality reduction for detecting gene-gene interactions in the presence of genotyping error, phenocopy and genetic heterogeneity. Genet Epidemiol 2003, 24:150-157.

25. Moore $\mathrm{J} \mathrm{H}$ : The ubiquitous nature of epistasis in determining susceptibility to common human diseases. Hum Hered 2003, 56:73-82.

26. Tong AH, Lesage G, Bader GD, Ding $H, X u H, X i n X$, Young J, Berriz GF, Brost RL, Change M, Chen Y, Cheng X, Chua G, Friesen H, Goldberg DS, Haynes J, Humphries C, He G, Hussein S, Ke L, Krogan N, Li Z, Levinson JN, Lu H, Menard P, Munyana C, Parsons AB, Ryan O, Tonikan R, Roberts T, Sdicu A, Shapiro J, Sheikh B, Suter B, Wong SL, Zhang LV, Zhu H, Gurd CG, Numro S, Sander C, Rine J, Greenblatt J, Peter M, Bretscher A, Bell G, Roth FP, Brown GW, Andrews B, Bussey $\mathrm{H}$, Boone $\mathrm{C}$ : Global mapping of the yeast genetic interaction network. Science 2004, 303:808-8I3.

27. Moore JH: A global view of epistasis. Nat Genet 2005, 37: I3-14.
28. Moore $J \mathrm{H}$, Williams SM: Traversing the conceptual divide between biological and statistical epistasis: Systems biology and a more modern synthesis. Bioessays 2005, 27:637-646.

29. Slonim DK: From patterns to pathways: gene expression data analysis comes of age. Nat Genet Suppl 2002, 32:502-508.

30. Mountain JL, Cavalli-Sforza LL: Multilocus genotypes, a tree of individuals, and human evolutionary history. Am J Hum Genet 1997, 61:705-718.

3I. Ritchie MD, White B, Parker JS, Hahn LW, Moore JH: Optimization of neural network architecture improves the power to identify gene-gene interaction in common diseases. BMC Bioinformatics 2003, 4:28.

32. Hauser ER, Watanabe RM, Duren WL, Bass MP, Langefeld CD, Boehnke M: Ordered subset analysis in genetic linkage mapping of complex traits. Genet Epidemiol 2004, 27:53-63.

33. Hoh J, Wille A, Ott J: Trimming, Weighting, and Grouping SNPs in Human Case-Control Association Studies. Genome Res 200I, II:2115-2119.

34. Ott J, Hoh J: Set association analysis of SNP case-control and microarray data. J Comput Biol 2003, 10:569-574.

35. Hahn LW, Ritchie MD, Moore JH: Multifactor dimensionality reduction software for detecting gene-gene and gene-environment interactions. Bioinformatics 2003, 19:376-382.

36. Hahn LW, Moore JH: Ideal discrimination of discrete clinical endpoints using multilocus genotypes. In Silico Biol 2004, 4:183-94.

37. Moore $\mathrm{JH}$ : Computational analysis of gene-gene interactions using multifactor dimensionality reduction. Expert Rev Mol Diagn 2004, 4:795-803.

38. Anderberg MR: Cluster Analysis for Applications New York: Academic Press; 1973.

39. Hanson R, Stutz J, Cheeseman P: Bayesian classification theory. In Technical Report \# FIA-90-12-7-0I Artificial Intelligence Research Branch, NASA Ames Research Center; 1991.

40. Han EH, Karypis G, Kumar V, Mobasher B: Clustering Based on Association Rule Hypergraphs. Proceedings of the SIGMOD Workshop on Research Issues on Data Mining and Knowledge Discovery: 1997; Tucson 1997:9-13.

41. Huang Z, Ng MK: A fuzzy k-modes algorithm for clustering categorical data. IEEE Trans Fuzzy Syst 1999, 7:446-452.

42. Hubert L, Arabie P: Comparing partitions. J Classif 1985, 2:193-218.

43. Han EH, Karypis G, Kumar V, Mobasher B: Clustering in High Dimensional Space Using Hypergraph Models. In Technical Report \#97-063 Computer Science and Engineering, University of Minnesota; 1997.

44. Duda RO, Hart PE: Pattern Classification and Scene Analysis New York: John Wiley and Sons; 1973.

45. Flegal KM, Carroll MD, Kuczmarski RJ: Overweight and obesity in the United States: prevalence and trends, 1960-1994. Int J Obe Relat Metab Disord 1998, 22:39-47.

46. Narod SA, Dupont A, Cusan L, Diamond P, Gomez J-L, Suburu R, Labrie F: The impact of family history on early detection of prostate cancer. Nat Med 1995, I:99-101.

47. Schultz S, Andreasen N: Schizophrenia. Lancet 1999, 353:1425-1430.

48. Kurtzke JF: Multiple sclerosis: changing times. Neuroepidemiology 1991, 10:1-8.

49. Li WT, Reich J: A complete enumeration and classification of two-locus disease models. Human Heredity 2000, 50:334-349.

50. Frankel WN, Schork NJ: Who's afraid of epistasis? Nat Genet 1996, 14:37|-373.

51. Kaufman L, Rousseeuw PJ: Finding Groups in Data: An Introduction to Cluster Analysis New York: John Wiley \& Sons, Inc; 1990.

52. Cheeseman P, Stutz J: Bayesian Classification (AutoClass): Theory and Results. In Advances in Knowledge Discovery and Data Mining Edited by: Fayyad UM, Piatetsky-Shapiro G, Smyth P, Uthurusamy R. Menlo Park: The AAAI Press; 1996.

53. Seno M, Karypis G: LPMiner: An Algorithm for Finding Frequent Itemsets Using Length-Decreasing Support Constraint. Proceedings of the IEEE Conference on Data Mining: 200 I; IEEE Computer Society 2001:505-512.

54. Steinley D: Properties of the Hubert-Arabie Adjusted Rand Index. Psychol Methods 2004, 9:386-396.

55. Good P: Permutation Tests: A Practical Guide to Resampling Methods for Testing Hypotheses New York: Springer; 2000. 